Conjugated Main Chain Azo-Polymers Based on Polycyclic Aromatic Hydrocarbons

\title{
Wiktorowicz, Szymon
}

2019-11

Wiktorowicz , S , Damlin , P , Salomäki , M , Kvarnström , C , Tenhu , H \& Aseyev , V 2019 ,

' Conjugated Main Chain Azo-Polymers Based on Polycyclic Aromatic Hydrocarbons ',

Macromolecular Chemistry and Physics , vol. 220 , no. 22 , 1900303 . https://doi.org/10.1002/macp.201900303

http://hdl.handle.net/10138/319903

https://doi.org/10.1002/macp.201900303

cc_by_nc

acceptedVersion

Downloaded from Helda, University of Helsinki institutional repository.

This is an electronic reprint of the original article.

This reprint may differ from the original in pagination and typographic detail.

Please cite the original version. 


\section{WILEY-VCH}

\section{Conjugated main chain azo-polymers based on polycyclic aromatic hydrocarbons}

Szymon Wiktorowicz*, Pia Damlin, Mikko Salomäki, Carita Kvarnström, Heikki Tenhu, and Vladimir Aseyev*

S. Wiktorowicz, Prof. H. Tenhu, V. Aseyev

Department of Chemistry

University of Helsinki

PB 55, A.I. Virtasen aukio 1, FIN-00014 HY, Finland

E-mail: szymon.wiktorowicz@vtt.fi, vladimir.aseyev@helsinki.fi

P. Damlin, M. Salomäki, Prof C. Kvarnström

Laboratory of Materials Chemistry and Chemical Analysis

Department of Chemistry

University of Turku

Vatselan-katu 2, FIN-20014 Turku, Finland

E-mail: carkva@utu.fi

Keywords: step-growth polymerization, azo polymers, polyaromatics, conjugated polymers

A reductive coupling reaction employing sodium bis(2-methoxyethoxy) aluminum hydride is used to prepare main chain azo-polymers comprising of polycyclic aromatic hydrocarbons (naphthalene, anthraquinone or fluorenone) from their dinitro-derivatives. The azo-bridges act as effective means of conjugation and all polymers exhibit differences in the UV-vis absorption and photoluminescence emission spectra depending on the degree of polymerization. Furthermore in the case of poly(azofluorenone)s and poly(azoanthraquinone)s these spectra may be modified by changes in the protonation state of the polymers. The LUMO and HOMO energy levels and the band gap of poly(azoanthraquinone) are estimated from cyclic voltammetry data and UV-Visible absorption of films. 


\section{WILEY-VCH}

\section{Introduction}

The development of conjugated and subsequently conductive polymers ${ }^{[1]}$ has revolutionized the field of polymer science by enabling the tailoring of materials with unique electronic and optical properties. The major structural component behind the design relies on the alternating double and single bonds present in the backbone of the polymer, which lead to overlap of the $\pi$-orbitals and a possible one-dimensional (1D) semi-conductive nature of the polymers. Upon doping, the band gap is decreased and the conductivity of these systems may further be enhanced towards a more metallic character. These properties have triggered the preparation of various new polymers which have found applications in organic electronics, ${ }^{[2]}$ solar cells, ${ }^{[3]}$ nanoparticles, ${ }^{[4]}$ chemo ${ }^{[5]}$ and biosensors, ${ }^{[6]}$ biomedical fields,,${ }^{[7]}$ etc.

Azobenzene has been extensively studied over the years ${ }^{[8]}$ predominantly in terms of its photo-induced, reversible trans-to-cis isomerization. Numerous azo-polymers have been reported in the past, with the azo-units in the main chain ${ }^{[9]}$ or as side groups. ${ }^{[10]}$ They have also been applied to more complicated architectures (block copolymers, ${ }^{[11]}$ dendrimers, ${ }^{[12]}$ cyclic $^{[13]}$ and supramolecular assemblies. ${ }^{[14]}$

As a result of the presence of a double bond in the azobenzene unit, it can be part of a $\pi$ conjugative system. In the past this strategy has received limited attention ${ }^{[15]}$ and majority of the studies deal with using rigid decoupled ${ }^{[16]}$ paraphenylene backbone to translate the structural changes upon trans-to-cis photo-isomerization ${ }^{[17]}$ into change of size. ${ }^{[18]}$ However, upon conjugation of the system, the photo-isomerization of the polymers is restricted. Recently, Telo et al. ${ }^{[19]}$ have prepared oligomeric azobenzenes and suggested that further development of poly(azobenzene) systems could result in good electronic conducting properties. 
In the past, we have developed multi-stimuli responsive main chain azo-polymers based on calix[4]arenes ${ }^{[20,21]}$ and dibenzo-18-crown-6-ethers. ${ }^{[21,22]}$ These polymers have been prepared from their corresponding nitro-derivatives in a one-step reductive coupling reaction using sodium bis(2-methoxyethoxy) aluminum hydride, Red-Al, in toluene.

Herein, we apply the synthetic strategy towards polycyclic aromatic hydrocarbons (naphthalene, anthraquinone, fluorenone) to produce multiple novel conjugated main chain azo-polymers (Scheme 1).

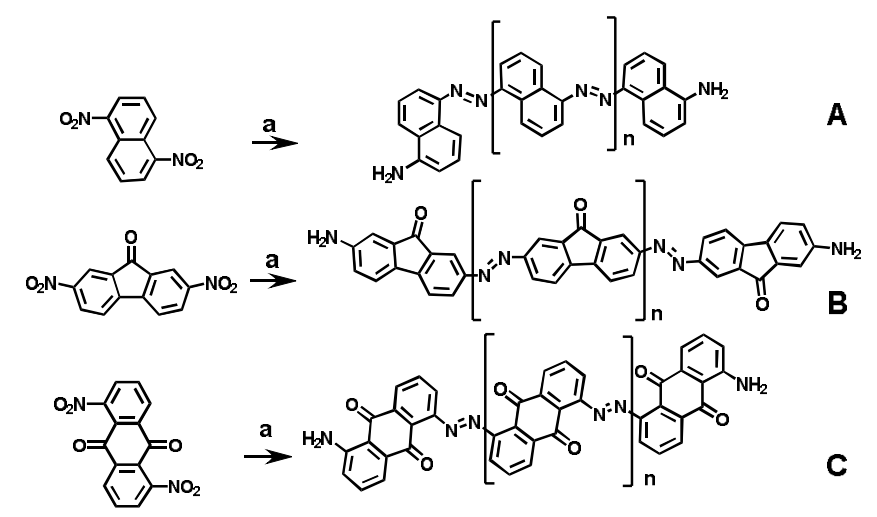

Scheme 1. Preparation of main chain azo-polymers in toluene using (a) Red-Al, sodium bis(2methoxyethoxy) aluminum hydride, $0^{\circ} \mathrm{C}$ : (A) poly(azonaphthalene), (B) poly(azofluorenone), and (C) poly(azoanthraquinone).

These polymers are shown to be soluble in polar organic solvents and fractionation of the crude reaction mixtures is possible. They exhibit dependence of absorption spectra on degree of polymerization, as well as in the case of poly(azofluorenone)s and poly(azoanthraquinone)s differences in emission and absorption spectra upon protonation/deprotonation. From drop casted films of poly(azoanthraquinone) cyclic voltammetry (CV) and optical spectroscopy 


\section{WILEY-VCH}

was applied to obtain the highest occupied molecular orbital (HOMO) and the lowest unoccupied molecular orbital (LUMO) energy levels of the polymers. ${ }^{[23]}$

In this report, the focus is on electrochemical properties of the new aromatic polymers. The presented approach of using nitro-derivatives directly towards preparation of main chain azopolymers provides an alternative pathway to the current oxidative coupling methods ${ }^{[24]}$ and is promising for the synthesis of other photo-active systems. The obtained molar mass distributions are broad, what is typical for a step growth polymerization. The highest molar mass fraction was isolated using chromatography in amounts sufficient for characterization. The more effective strategy to synthesis of reported polymers and their isolation will be a subject of a separate publication.

\section{Results}

The reductive coupling reactions employing sodium bis(2-methoxyethoxy) aluminum hydride, Red-Al were done in toluene at $0^{\circ} \mathrm{C}$ using commercially available monomers $(1,5-$ dinitronaphthalene, 2,7-dinitro-9-fluorenone, 1,5-dinitroanthraquinone) at high concentrations (50-100 g/L). The formation of the azo-bridge was primarily evaluated on an 4,4'dimethoxyazobenzene model compound to determine the experimental conditions for the highest efficiency (SI, Figure S1-S2, Table S1). The reaction occurs with a side product of 4aminoanisole in minor trace. The best yield for the azo-product $(71 \%)$ is obtained for reactions, which utilize 3 equivalents of Red-Al per 1 nitro group. This enhancement in reaction conditions was then applied to coupling of dinitro-derivatives and upon work up of crude reaction mixtures, which were analyzed with the size exclusion chromatography (SEC) (SI, Figure S3) the development of larger molecular weights was evident. This reaction being 


\section{WILEY-VCH}

a step-growth polymerization, has the inevitable characteristic of broad molecular weight distribution and an effort to isolate fractions of lower polydispersity needed to be done. When the polymers were solubilized in a good solvent (THF) at high concentrations and small amount of non-solvent (n-hexane) was added, the resulting precipitant upon collection (centrifugation) was analyzed to reveal improved polydispersity values. The procedure was then repeated until satisfactory results could be obtained (Figure 1). For all the polymers, selected fractions were isolated, the molecular weight characteristics of which (SI, Table S2, Figure S4 and S5) indicate degrees of polymerization up to 30 and polydispersity indexes between 1.4 and 2.6.

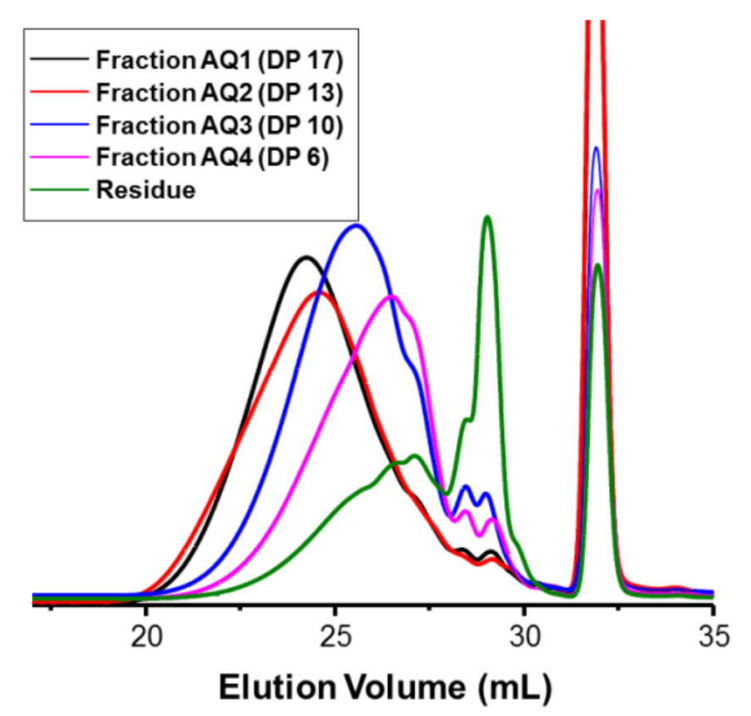

Figure 1. Example of fractionation of the polymers: comparison of the SEC eluograms (THF) obtained using RI-detector for Fractions AQ1-4 of poly(azoanthraquinone)s and the remaining residue.

In order to evaluate the structure of the polymers, comparative FTIR measurements were performed (SI, Figure S6, S7, and S8). Upon coupling, the asymmetric and symmetric stretching bands for the dinitro-monomers at around 1520 and $1340 \mathrm{~cm}^{-1}\left(\mathrm{~N}=\mathrm{O}\right.$ of $\left.\mathrm{NO}_{2}\right)$ disappear and a strong band around $1600 \mathrm{~cm}^{-1}$ due to $\mathrm{N}=\mathrm{N}$ stretching emerges $\left(1577 \mathrm{~cm}^{-1}\right.$ for 


\section{WILEY-VCH}

poly(azonapthalene)s, $1596 \mathrm{~cm}^{-1}$ for poly(azofluorenone)s and $1600 \mathrm{~cm}^{-1}$ for

poly(azoanthraquinone)s) indicating the successful formation of the azo-bridge.

In the case of ${ }^{1} \mathrm{H}$ NMR spectra $\left(500 \mathrm{MHz}, \mathrm{DMSO}-\mathrm{d}_{6}\right)$, the polymers give broad signals in the aromatic region $(6.5-8.5 \mathrm{ppm})$ rendering the analysis difficult. This may be attributed to the polymeric nature of the systems (reduced mobility), conjugation, as well as possible conformational isomers. Certain regions of the spectra may be distinguished (SI, Figure S9, S10, and S11) as the protons attached to the ortho and para positions with regard to the azobridge will experience a downfield shift $(>7.7 \mathrm{ppm})$. Furthermore, the signals for the protons in the meta position (for both the amino and azo functionalities) will appear between 7.0-7.7 ppm. Also, the up field shifted signals $(6.5-7.0 \mathrm{ppm})$ correspond to the protons adjacent to the meta positions to the amino groups. When comparing the ${ }^{1} \mathrm{H}$ NMR spectra of the polymers to oligomeric fractions, we see better spectral definition in the latter case and an increase in intensity of signals corresponding to the protons adjacent to amino groups. This is not surprising considering that the occurrence of the amino end groups in the oligomers is larger.

The analysis of ${ }^{13} \mathrm{CNMR}$ spectra of the oligomers further supports the formation of the azolinkage (SI, Figure S12, S13, and 14). For poly(azonaphthalene)s the carbon attached to the azo-moiety appears at $149.5 \mathrm{ppm}$, while for the amino group at $144 \mathrm{ppm}$. In the case of poly(azofluorenone)s those signals appear at 151 and $147 \mathrm{ppm}$ respectively and for poly(azoanthraquinone)s they are shifted to 152.5 and $146.5 \mathrm{ppm}$. The remaining signals are in accord with the proposed structure.

In a subsequent study, we evaluate the absorption properties of the polymers in solution. UVVis spectra in THF (or 5\% DMF in THF) for different polymer fractions with varying degree of polymerization, DP were recorded (Figure 2). 


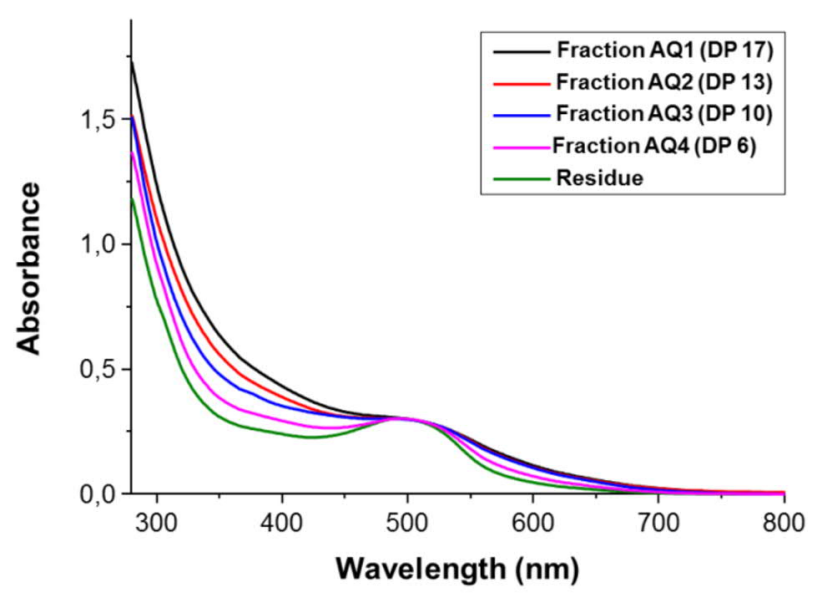

Figure 2. UV-Vis spectra of poly(azoanthraquinone) fractions AQ1-4 (0.1 g/L in THF) depicting the dependence of absorbance on the degree of polymerization.

In the case of poly(azoanthraquinone)s, with increase in the chain length, the absorption spectrum of the polymers becomes broader (300-700nm) with increasing absorption in the $300-450 \mathrm{~nm}$ region. The distinct maximum observed for oligomers $(\mathrm{DP}<6)$ at $500 \mathrm{~nm}$ becomes less obvious for the polymers. The larger polymer fractions have a greater conjugation length and electron transitions are possible over a broader range of wavelengths. This trend may also be observed for the poly(azonaphthalene)s (SI, Figure S15) and poly(azofluorenone)s (SI, Figure S16), indicating that for all the azo-polymers the absorption depends on the degree of polymerisation. Similar dependence is indicated for the photoluminescence, PL emission spectra of the polymers (Figure 3). The polymers were excited at different wavelengths (SI, Figure S17 and S18) and the fractions with highest and lowest DP compared. A general tendency being that the lower extent of polymerization results in better emission. 


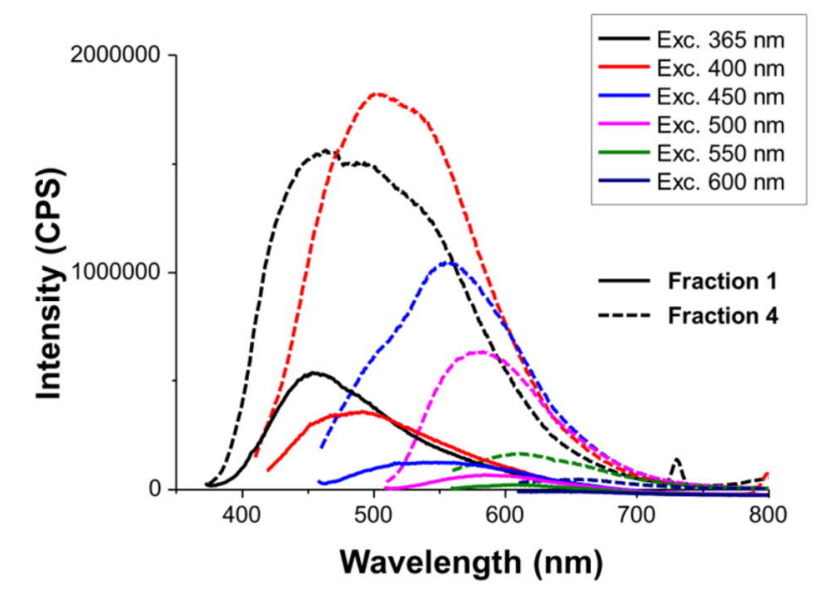

Figure 3. Dependence of emission on degree of polymerisation of poly(azoanthraquinone) (Fraction 1 and Fraction 4) in 5\% DMF in THF (c=0.1 g/L).

Owing to the carbonyl groups present in the structure, poly(azoanthraquinone)s and poly(azofluorenone)s may be reversibly protonated. We wanted to see how this influences the spectral properties. Both absorption and PL emission spectra were recorded. The addition of minimal amount of acid does not significantly alter the absorption spectrum for the poly(azoanthraquinone)s (Figure 4a). However, the emission is significantly decreased (Figure 4b), while an opposite effect can be observed for the fluorenones (SI, Figures S19 and S20). With shorter chains, the protonation of poly(azofluorenone)s leads to a $50 \mathrm{~nm}$ hypsochromic shift of the maximum at $450 \mathrm{~nm}$. The absorption still occurs in a broad range of wavelengths indicating the efficient conjugation. It is noteworthy, that despite elongated irradiation times and varying wavelengths, no trans-to-cis isomerization of the azo-bridge could be induced for all studied polymers in solvents (SI, Figure S21). 

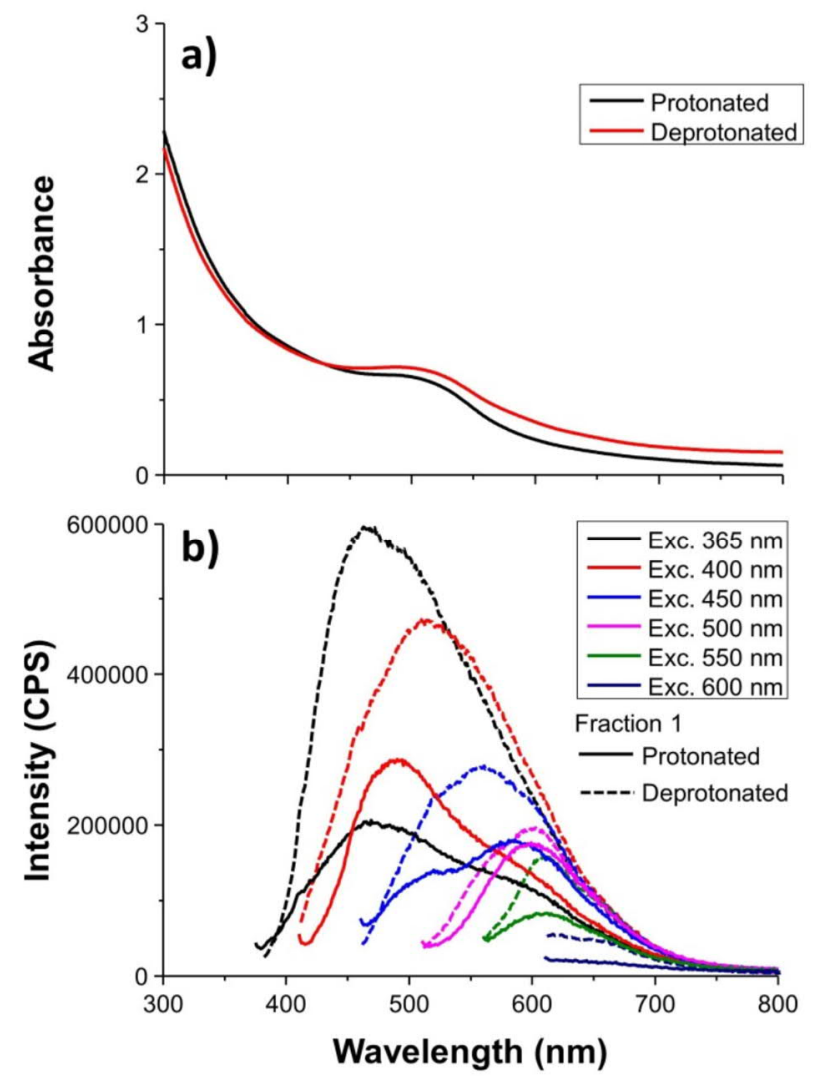

Figure 4. Influence of protonation state on absorbance (a) and emission (b) of the poly(azoanthraquinone)s in 5\% DMF in THF (c=0.15 g/L).

Cyclic voltammetry $(\mathrm{CV})$ is an electrochemical method commonly used in polymer research to investigate the electrochemical behavior as well as to assess the position of both HOMO and LUMO energy levels. ${ }^{[23]}$ Figure 5 shows cyclic voltammograms of monomeric dinitroanthraquinone in acetonitrile solution and drop casted poly(azoanthraquinone) on glassy carbon electrode during reduction. The monomer CV shows that the anthraquinone develops two quasi-reversible one-electron reductions at $\mathrm{E}^{\mathrm{Red}}{ }_{1 / 2}=-0.53 \mathrm{~V}$ and $-1.18 \mathrm{~V}$. The first redox couple corresponds to formation of radical anions, and the second couple relates to formation of dianions. The reduction process at $-1.02 \mathrm{~V}$ is the product of irreversible reduction of nitro substituents. Analogous electrochemical nitro group reduction in 1nitroanthraquinone have been observed earlier. ${ }^{[25]}$ The azo-group reduction is not observed at measured potential range. It expected to take place at higher cathodic potentials. ${ }^{[19]}$ The redox 


\section{WILEY-VCH}

couple separation in anthraquinones is usually in the range of 0.5 to $0.7 \mathrm{~V}$ depending on the substituents. ${ }^{[26,27]}$ The CV of poly(azoanthraquinone) shows two electron reduction in a combined cathodic wave. On the other hand, oxidation produces two waves with ca. $0.1 \mathrm{~V}$ separation. The voltammogram remains essentially constant over several cycles. The combination and broadening of reduction waves is considered as an indication of electron delocalization along the polymer chain. ${ }^{[27]}$ The onset of reduction of poly(azoanthraquinone) takes place at higher cathodic voltages comparing to corresponding monomer. There are some factors that might delay the electrochemical reduction in the polymer film. While the monomer is in solution, the polymer has probably a highly $\pi$-stacked conformation on the electrode surface and the utilized electrolyte $\left(\mathrm{TEABF}_{6}\right)$ is quite bulky possessing a reasonably slow migration inside the film during the electrochemical reduction.

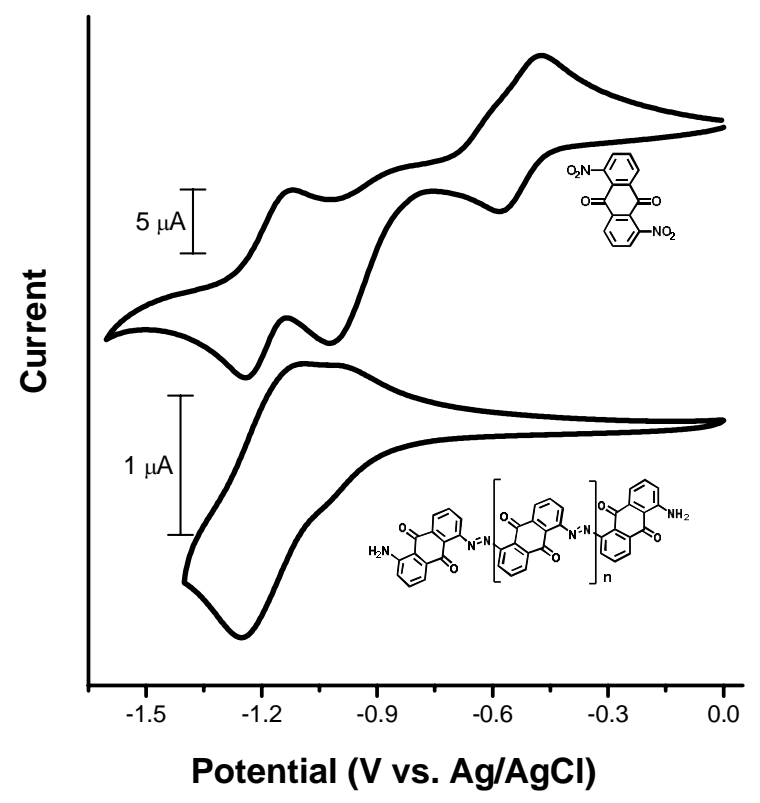

Figure 5. Cyclic voltammograms recorded from monomeric dinitroanthraquinone $2 \mathrm{mM}$ in acetonitrile and drop-casted poly(azoanthraquinone) film on glassy carbon electrode in acetonitrile $\left(0.1 \mathrm{M} \mathrm{TEABF}_{6}\right)$. Scan rate $50 \mathrm{mVs}^{-1}$. 


\section{WILEY-VCH}

From $\mathrm{CV}$ the onset potential for reduction $\left(\mathrm{E}^{\mathrm{red}}{ }_{\text {onset }}\right)$ was found at $-0.8 \mathrm{~V}$. The reduction potential was determined from the onset of the curve by taking the intercept between the line tangent to the baseline and the line tangent to the point of the largest slope value. From this potential value the corresponding LUMO level or electron affinity level could be determined using the empirical relationship proposed by Brédas et al. using a scale factor of $4.4 \mathrm{eV}$ relating $\mathrm{Ag} / \mathrm{AgCl}$ to vacuum ${ }^{[28]}$ giving a value of $-3.63 \mathrm{eV}$.

The absorption edge of the UV-Vis spectra shown for a poly(azoanthraquinone) film in SI, Figure S22 is used for estimating the optical bandgap of approx. $1.9 \mathrm{eV}$ which gives a value of $-5.53 \mathrm{eV}$ for HOMO energy level. The maximum absorbance is found at $520 \mathrm{~nm}$, which is slightly blue shifted in comparison to the solution phase spectrum in Figure 2. Bandgaps calculated from electrochemical potentials for redox conversion have often been found to be higher than the optical bandgaps. The broader absorption peak in films means that there is a distribution of energy levels corresponding to the $\pi$ - $\pi^{*}$ transitions.

To obtain more information on the electrochemical reduction process, visible range spectroelectrochemical measurement was conducted on a drop-casted poly(azoanthraquinone) film on FTO-glass electrode. Figure 6 shows differential spectra of the film upon reduction. Stating from - $0.6 \mathrm{~V}$ a broad absorbance increase near $500 \mathrm{~nm}$ starts to develop. At the potential of $-0.7 \mathrm{~V}$ there are clear bands near 400 and $500 \mathrm{~nm}$ and a weak shoulder at ca. 550 $\mathrm{nm}$. The latter is a characteristic band attributed to radical anion. ${ }^{[27,29,30]}$ However, the shoulder disappears upon further reduction and the bands near 400 and $500 \mathrm{~nm}$ continue to develop, which is an indication of very short lived radical cation. The band near $500 \mathrm{~nm}$ is slightly red shifted to $477 \mathrm{~nm}$ during the reduction and is characteristic for the dianion. Slightly sharper band at near $400 \mathrm{~nm}$ is blue shifted to $416 \mathrm{~nm}$ and is accredited to a protonated dianion. ${ }^{[27,29,30]}$ The apparent protonation must be due to traces of water present in 


\section{WILEY-VCH}

the solvent. The protonation in organic solvents has been discussed earlier. ${ }^{[29,30]}$ The protonated form of dianion has higher molar absorption therefore the level of protonation is difficult to estimate. Increase of absorbance is also observed at near $300 \mathrm{~nm}$, which is related anthraquinone reduction, but it cannot be quantified because of restrictions given by the glass substrate. The appearance of dianion bands with only minor reference to radical anion band is again strong indication of effective electron delocalization, the same phenomena as realized in single reduction wave in the $\mathrm{CV}$. For the reason that the spectra were measured as a steady state conditions the bands start to develop earlier comparing to electrochemical processes observed by cyclic voltammetry. A wide band above $600 \mathrm{~nm}$ is also accredited to dianion species. Furthermore, indication of extended electron delocalization is seen in the appearing bands at 720 and $790 \mathrm{~nm}^{\text {[27] }}$

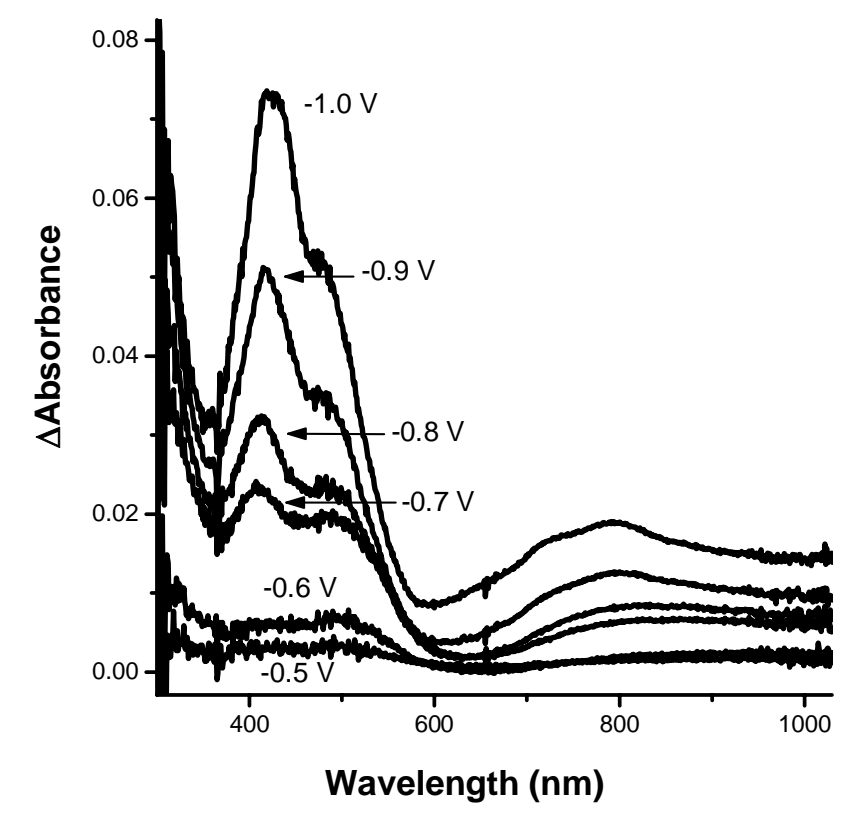

Figure 6. Spectra of reduction of drop casted poly(azoanthraquinone) on FTO electrode. The spectrum at $-0.4 \mathrm{~V}$ vs. $\mathrm{Ag} / \mathrm{AgCl}$ is taken as reference. 


\section{WILEY-VCH}

\section{Conclusion}

The reported reductive coupling reaction offers a versatile pathway towards the preparation of main chain azo-polymers (based on naphthalene, fluorenone, anthraquinone) from dinitromonomers, as opposed to the oxidative coupling routes which utilize reduced aniline counterparts. We have used this approach towards the preparation of novel polymers based on polycyclic aromatic hydrocarbons, by using the azo-bridge as a means of conjugation. All the organo-soluble polymers have been subjected to simple fractionation protocols and the structure is elucidated with FTIR and NMR analysis. Absorption and photoluminescence characterization indicates a strong dependence of spectra on the degree of polymerization. The intensity of PL emission may furthermore be manipulated by the protonation state of the poly(azoanthraquinone)s and poly(azofluorenone)s. These n-type semiconductor materials with band gaps of ca $1.9 \mathrm{eV}$ can act as counterpart for the different p-type materials available and utilized in optical devices.

Additionally, we believe that the described facile method may further contribute towards the development of new and exciting photoactive materials including photo-actuators, sensors, redox active systems and conductive polymers.

\section{Experimental Section}

All starting compounds for polymerisation were of reagent grade quality and purchased directly from commercial suppliers. Solvents were dried by means of molecular sieves prior to reaction. Sodium bis(2-methoxyethoxy) aluminium hydride, Red-Al was purchased from Aldrich as $60 \mathrm{wt} \%$ solution in toluene. 


\section{WILEY-VCH}

General Procedures for Preparation of Polymers and Model Compound: The dinitro-PAH derivative (1,5-dinitronaphthalene, 2,7-dinitro-9-fluorenone, 1,5-dinitro-9,10-anthraquinone) or 4-nitroanisole (Model Compound precursor -see Table S1) was suspended in toluene (0.1 $\mathrm{g} / \mathrm{mL}$ ) and added dropwise to a cooled solution of Red-Al (60 wt $\%$ in toluene, 6 eq.). The resulting mixture was stirred under an argon atmosphere at room temperature for 2-5 days upon which minimal amount of methanol was added to quench the reaction (caution! exothermic reaction) and the evolution of gas subsided. Residue was taken up with $10 \% \mathrm{HCl}$ (neutralization) and extracted with chloroform. A solid residue from the interphase was then washed with THF and subsequently with DMF. The combined organic phase was evaporated to give the crude reaction mixture, which was analyzed with SEC. In case of the model compound column chromatography was done to yield the azoanisole product (Ethyl acetate:hexane).

Fractionation of the Polymers: Concentrated solutions of the Polymers in THF (25 g/L) were left stirring overnight at elevated temperatures $\left(50^{\circ} \mathrm{C}\right)$ to ensure best solubilization. Subsequently, small amount of hexane was added to the mixtures until the appearance of a cloud point. The suspension was left stirring for $1 \mathrm{~h}$ and the precipitate collected by means of a centrifuge. In a following step more hexane was added to the system and the procedure was continued until no cloud point could be observed upon addition of a large amount of hexane. In cases when polydispersity of the fractions was high, a re-fractionation was performed.

Size Exclusion Chromatography (SEC) for the studied samples was done in DMF or THF using a Waters instrument which was equipped with Waters Styragel HR6, HR4 and HR2 columns (7.8 x $300 \mathrm{~mm}$ each), monitored with Waters $2410 \mathrm{RI}$ or Waters $2487 \mathrm{UV}$ (monitoring at $230 \mathrm{~nm}$ ) detectors with a flow rate of $0.8 \mathrm{~mL} / \mathrm{min}$ and referenced against 


\section{WILEY-VCH}

poly(styrene) standards (Scientific Polymer Products Inc.) or poly(methyl methacrylate) from Polymer Standards Service-USA Inc..

Fourier Transform Infrared Spectroscopy (FTIR) measurements were done using a PerkinElmer Spectrum One FTIR Spectrometer in the solid state.

Ultra-Violet-Visible Light (UV-Vis) spectra for all fractionated samples were obtained with a Shimadzu 2501 PC spectrometer in the range of 280-800 nm. Sample concentration was kept the same $(0.1 \mathrm{~g} / \mathrm{L})$ and the measurements were done in THF, DMF and DMSO.

Emission spectra were recorded using a Fluoromax-4 Spectrofluorometer from HoribaJobin Yvon (exit slit: $2 \mathrm{~nm}$, entrance slit: $2 \mathrm{~nm}$, varying scanning range) on the samples prepared for $\mathrm{UV}-\mathrm{V}$ is measurements.

${ }^{1} H$ NMR and ${ }^{13} C$ NMR spectra were obtained with a Bruker Avance III 500MHz spectrometer in deuterated dimethyl sulfoxide (DMSO) or chloroform.

The Cyclic Voltammetric ( $\mathrm{CV}$ ) measurements were conducted with an Autolab potentiostat controlled by the Nova 1.10 software. A glassy carbon electrode was used as working electrode (WE), a platinum electrode as counter electrode (CE) and a silver electrode as reference electrode (RE). Tetraethylammonium hexafluorophosphate, $0.1 \mathrm{M}$ in acetonitrile was used as electrolyte. 


\section{WILEY-VCH}

\section{Supporting Information}

Supporting Information is available from the Wiley Online Library or from the author. It includes materials characterization: SEC traces, FTIR, 1H and 13C NMR spectra, UV-Vis and photoluminescence experiments, and the molar mass characteristics.

\section{Acknowledgements}

The financial support of the Academy of Finland (project number 260486) is gratefully acknowledged.

\section{Conflict of Interest}

The authors declare no conflict of interest.

Received: ((will be filled in by the editorial staff))

Revised: ((will be filled in by the editorial staff)) Published online: ((will be filled in by the editorial staff)) 
Main chain azo-polymers comprising of polycyclic aromatic hydrocarbons (naphthalene, anthraquinone or fluorenone) are synthesized from corresponding dinitro-derivatives as repeating units. The azo-bridges act as effective means of conjugation. These n-type semiconductor materials may find use as counterparts for the available p-type materials and utilized in further development of new organic electronics.

Keyword: main chain azo-polymers

S. Wiktorowicz*, P. Damlin, M. Salomäki, C. Kvarnström, H. Tenhu, V. Aseyev*

Conjugated main chain azo-polymers based on polycyclic aromatic hydrocarbons

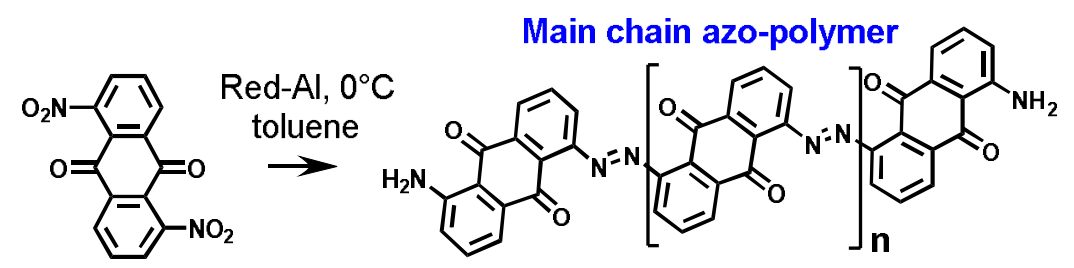




\section{WILEY-VCH}

References

[1] H. Shirakawa, E. J. Louis, A. G. MacDiarmid, C. K. Chiang, A. J. Heeger, J. Chem. Soc., Chem. Comm. 1977, 578.

[2] A. Facchetti, Chem. Mater. 2010, 23, 733.

[3] a) X. Zhan, D. Zhu, Polym. Chem. 2010, 1, 409; b) H. Zhou, L. Yang, W. You, Macromolecules 2012, 45, 607.

[4] J. Pecher, S. Mecking, Chem. Rev. 2010, 110, 6260.

[5] a) H. N. Kim, Z. Guo, W. Zhu, J. Yoon, H. Tian, Chem. Soc. Rev. 2011, 40, 79; b) S. W. Thomas, G. D. Joly, T. M. Swager, Chem. Rev. 2007, 107, 1339.

[6] K. Li, B. Liu, Polym. Chem. 2010, 1, 252.

[7] C. Zhu, L. Liu, Q. Yang, F. Lv, S. Wang, Chem. Rev. 2012, 112, 4687.

[8] H. M. D. Bandara, S. C. Burdette, Chem. Soc. Rev. 2012, 41, 1809.

[9] A. Natansohn, P. Rochon, Chem. Rev. 2002, 102, 4139.

[10] D. Wang, X. Wang, Prog. Polym. Sci. 2013, 38, 271.

[11] Y. Zhao, J. Mater. Chem. 2009, 19, 4887.

[12] R. Deloncle, A.-M. Caminade, J. Photochem. Photobiol. C: Photochem. Rev. 2010, 11, 25.

[13] X. Xu, N. Zhou, J. Zhu, Y. Tu, Z. Zhang, Z. Cheng, X. Zhu, Macromol. Rapid Commun. 2010, 31, 1791.

[14] a) X. Yan, F. Wang, B. Zheng, F. Huang, Chem. Soc. Rev. 2012, 41, 6042; b) R. Dong, Y. Liu, Y. Zhou, D. Yan, X. Zhu, Polym. Chem. 2011, 2, 2771.

[15] a) M. E. Wright, M. Porsch, J. Polym. Prepr. 1998, 39, 278; b) A. Izumi, M. Teraguchi, R. Nomura, T. Masuda, Macromolecules 2000, 33, 5347; c) A. Izumi, M. Teraguchi, R. Nomura, T. Masuda, J. Polym. Sci. A: Polym. Chem. 2000, 38, 1057.

[16] D. Bléger, J. Dokić, M. V. Peters, L. Grubert, P. Saalfrank, S. Hecht, J. Phys. Chem. B 2011, 115, 9930.

[17] A. G. Tennyson, B. Norris, C. W. Bielawski, Macromolecules 2010, 43, 6923.

[18] D. Bléger, T. Liebig, R. Thiermann, M. Maskos, J. P. Rabe, S. Hecht, Angew. Chem. Int. Ed. 2011, 50, 12559.

[19] Á. Moneo, G. C. Justino, M. F. N. N. Carvalho, M. C. Oliveira, A. M. M. Antunes, D. Bléger, S. Hecht, J. P. Telo, J. Phys. Chem. A, 2013, 117, 14056. 
[20] a) S. Wiktorowicz, V. Aseyev, H. Tenhu, Polym. Chem. 2012, 3, 1126; b) S. Wiktorowicz, H. Tenhu, V. Aseyev, Polym. Chem. 2013, 4, 2898; c) S. Wiktorowicz, H. Tenhu, V. Aseyev, Macromolecules 2013, 46, 6209.

[21] S. Wiktorowicz, H. Tenhu, V. Aseyev, in Temperature-Responsive Polymers: Chemistry, Properties and Applications, (Eds: V. V. Khutoryanskiy, T. K. Georgiou), Wiley 2018, Ch. 6: Multi-stimuli-responsive Polymers Based on Calix[4]arenes and Dibenzo-18crown-6-ethers, pp 145-176.

[22] S. Wiktorowicz, R. Duchene, H. Tenhu, V. Aseyev, Polym. Chem. 2014, 5, 4693.

[23] T. Johansson, W. Mammo, M. Svensson, M. R. Andersson, O. Inganäs, J. Mater. Chem. 2003, 13, 1316.

[24] a) H. T. Nguyen, O. Coulembier, K. Gheysen, J. C. Martins, P. Dubois, Macromolecules 2012, 45, 9547; b) C. Zhang, N. Jiao, Angew. Chem. Int. Ed. 2010, 49, 6174.

[25] H. Gunasingham, B.T. Tay, K.P. Ang, Anal. Chem. 1987, 59, 262.

[26] P. H. Given, M. E. Peover, J. Chem. Soc. 1960, 385.

[27] T. Yamamoto, H. Etori, Macromolecules 1995, 28, 3371.

[28] J. L. Brédas, R. Silbey, D. S. Boudreaux, R. R. Chance, J. Amer. Chem. Soc. 1983, 105, 6555.

[29] M. Shamsipur, B. Hemmateenejad, A. Babaei, L. Faraj-Sharabiani J. Electroanal. Chem. 2004, 570, 227.

[30] A. Babaei, P. A. Connor, A. J. McQuillan, S. Umapathy, J. Chem. Educ. 1997, 74, 1200. 
Copyright WILEY-VCH Verlag GmbH \& Co. KGaA, 69469 Weinheim, Germany, 2018.

Supporting Information

\section{Conjugated main chain azo-polymers based on polycyclic aromatic hydrocarbons}

Szymon Wiktorowicz ${ }^{\dagger *}$, Pia Damlin ${ }^{\ddagger}$, Mikko Salomäki ${ }^{\ddagger}$, Carita Kvarnström ${ }^{\ddagger}$, Heikki Tenhu ${ }^{\dagger}$ and Vladimir Aseyev ${ }^{\dagger *}$

${ }^{\dagger}$ University of Helsinki, A.I. Virtasen aukio 1, PB 55, FIN-00014, HY, Finland

${ }^{\ddagger}$ Laboratory of Materials Chemistry and Chemical Analysis, Department of Chemistry, University of Turku, Vatselankatu 2, FIN-20014 Turku, Finland

\section{Characterisation}<smiles>COc1ccc(N=Nc2ccc(OC)cc2)cc1</smiles>

Fig. S1 Preparation of model compound (4,4'-dimethoxyazobenzene) with possible side product

Table S1 Adjusting of coupling conditions for 4-nitroanisole

\begin{tabular}{|c|c|c|c|c|c|c|c|c|}
\hline & Reagent & $\begin{array}{c}\text { T } \\
\left({ }^{\circ} \mathbf{C}\right)\end{array}$ & Addition & $\begin{array}{c}\text { Ratio (Red- } \\
\text { Al/reagent) }\end{array}$ & Solvent & $\begin{array}{c}\text { Concentration } \\
(\mathbf{M})\end{array}$ & $\begin{array}{c}\text { Time } \\
(\mathbf{h})\end{array}$ & Result $^{\mathbf{1}}$ \\
\hline 1 & NPhOMe & $0-\mathrm{RT}$ & Fast & $3: 1$ & Toluene & 1.6 & 0.75 & AzPhOMe $(71 \%)$ \\
\hline 2 & NPhOMe & $0-\mathrm{RT}$ & Fast & $2: 1$ & Toluene & 1.6 & 0.75 & AzPhOMe $(55 \%)$ \\
\hline 3 & NPhOMe & $0-\mathrm{RT}$ & Fast & $1.5: 1$ & Toluene & 1.6 & 0.75 & $\mathrm{AzPhOMe}(40 \%)$ \\
\hline 4 & NPhOMe & $0-\mathrm{RT}$ & slow & $3: 1$ & Toluene & 1.6 & 0.75 & AzPhOMe $(68 \%)$ \\
\hline
\end{tabular}

$\mathrm{NPhOMe} \mathrm{-} \mathrm{4-nitroanisole,} \mathrm{AzPhOMe} \mathrm{-} \mathrm{4,4'-dimethoxyazobenzene,}$

Result $^{1}$ - Obtained from gravimetric analysis of column chromatography fractions (compared to amount of starting compound). Slow addition: 1 drop every 5-10s. Fast addition: steady, drop by drop. 
WILEY-VCH<smiles>COc1ccc(/N=N/c2ccc(OC)cc2)cc1</smiles>

c

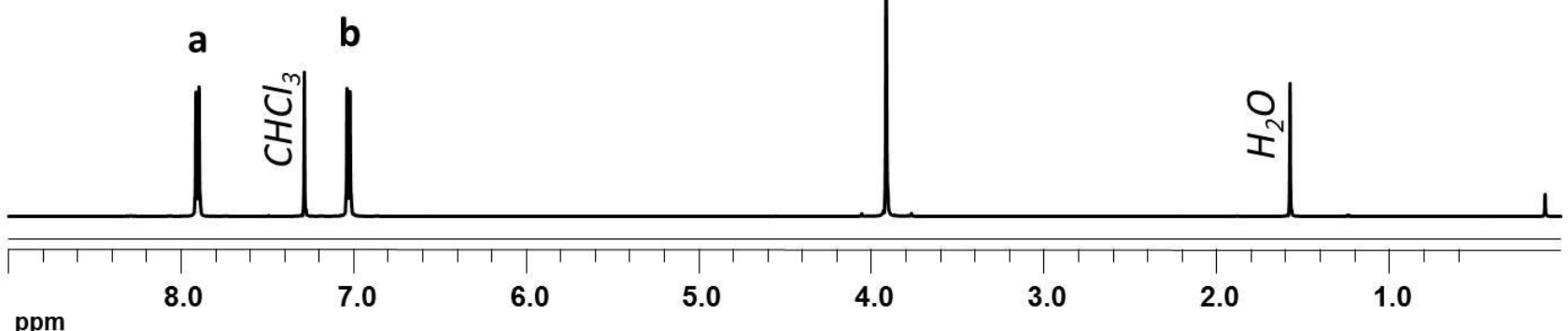

Fig. S2 Structure and ${ }^{1} \mathrm{H}$ NMR spectrum of $4,4^{\prime}$-dimethoxyazobenzene in $\mathrm{CDCl}_{3}$ obtained from coupling reaction

4,4'-dimethoxyazobenzene: ${ }^{1} \mathrm{H}$ NMR $\left(500 \mathrm{MHz}, \mathrm{CDCl}_{3}\right): \delta=3.92\left(\mathrm{~s}, \mathrm{OCH}_{3}, 6 \mathrm{H}\right), 7.03(\mathrm{~d}, \mathrm{Ar} H \mathrm{~m}-\mathrm{N}=\mathrm{N}$, 4H), $7.9(\mathrm{~d}, \mathrm{Ar} H o-\mathrm{N}=\mathrm{N}, 4 \mathrm{H}) .{ }^{13} \mathrm{C} \mathrm{NMR}\left(125 \mathrm{MHz}, \mathrm{CDCl}_{3}\right): \delta=161.3,144.1,124.2,115.0,56.2$. FTIR: $v_{\max }$ $\left(\mathrm{cm}^{-1}\right): 1594(\mathrm{~N}=\mathrm{N})$

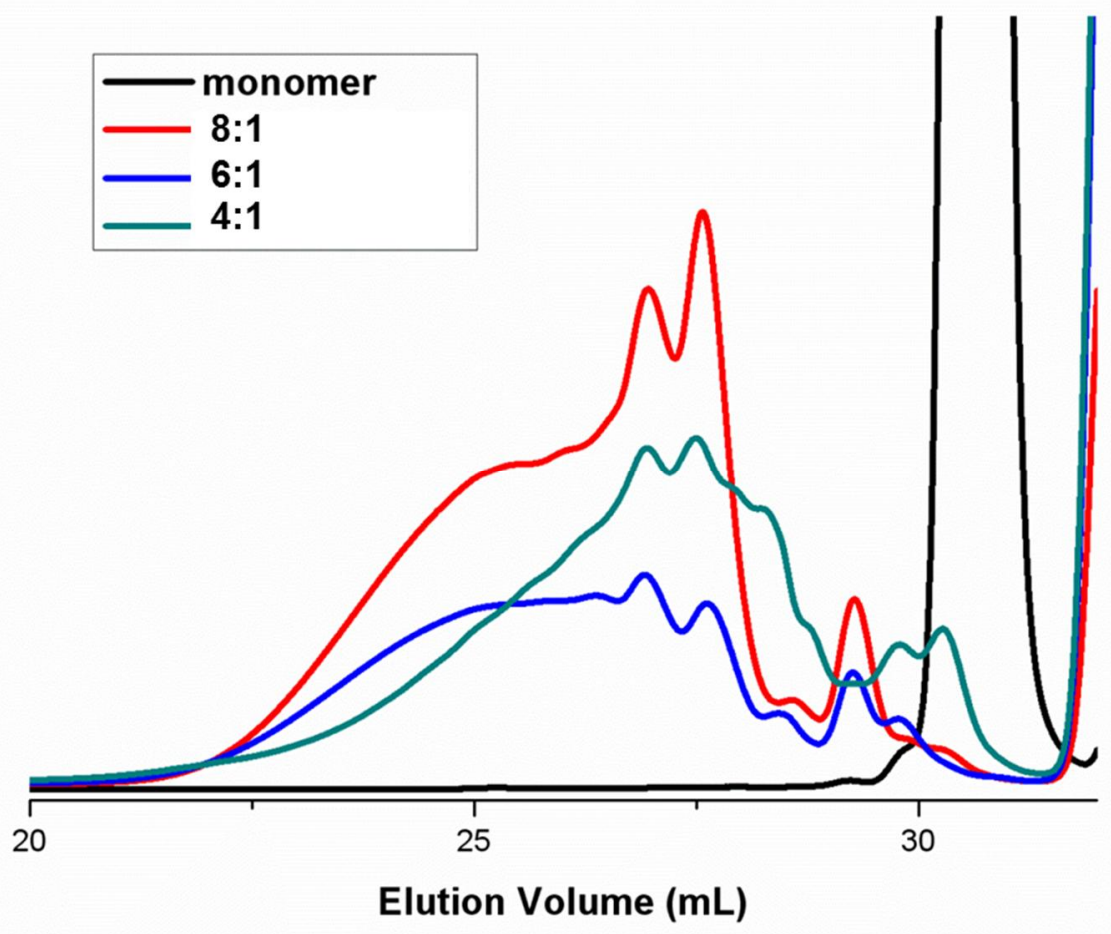

Fig. S3 Adjusting of coupling conditions for poly(azonaphthalene)s. SEC traces (THF) of crude reaction mixtures from reactions with varying Red-Al:monomer ratios. 
Table S2 Molecular weight characteristics of selected fractions of Polymers

Poly(azonaphthalene)s

\begin{tabular}{|c|c|c|c|c|c|}
\hline & Name & $\mathbf{M}_{\mathbf{w}, \mathbf{G P C}}$ & $\mathbf{M}_{\mathbf{n}, \mathbf{G P C}}$ & $\mathbf{P D I}$ & $\mathbf{D P}_{\mathbf{w}}$ \\
\hline $\mathbf{1}$ & $\mathbf{A N 1}$ & 4600 & 1800 & 2.55 & 30 \\
\hline $\mathbf{2}$ & $\mathbf{A N 2}$ & 3100 & 1500 & 2.1 & 20 \\
\hline $\mathbf{3}$ & $\mathbf{A N 3}$ & 2800 & 1450 & 1.9 & 17 \\
\hline $\mathbf{4}$ & $\mathbf{A N 4}$ & 2100 & 1250 & 1.7 & 14 \\
\hline $\mathbf{5}$ & $\mathbf{A N 5}$ & 1900 & 1200 & 1.6 & 12 \\
\hline $\mathbf{6}$ & AN6 & 1200 & 850 & 1.4 & $<7$ \\
\hline $\mathbf{7}$ & AN7 & 850 & 600 & 1.5 & $<5$ \\
\hline
\end{tabular}

Poly(azofluorenone)s

\begin{tabular}{|c|c|c|c|c|c|}
\hline & Name & $\mathbf{M}_{\mathbf{w}, \mathbf{G P C}}$ & $\mathbf{M}_{\mathbf{n}, \mathbf{G P C}}$ & $\mathbf{P D I}$ & $\mathbf{D P}_{\mathbf{w}}$ \\
\hline $\mathbf{1}$ & $\mathbf{A F 1}$ & 3500 & 1350 & 2.6 & 17 \\
\hline $\mathbf{2}$ & $\mathbf{A F 2}$ & 3300 & 1300 & 2.4 & 16 \\
\hline $\mathbf{3}$ & $\mathbf{A F 3}$ & 2700 & 1250 & 2.1 & 13 \\
\hline $\mathbf{4}$ & $\mathbf{A F 4}$ & 2300 & 1200 & 1.9 & 11 \\
\hline $\mathbf{5}$ & $\mathbf{A F 5}$ & 2100 & 1150 & 1.8 & 10 \\
\hline $\mathbf{6}$ & $\mathbf{A F 6}$ & 1800 & 1100 & 1.6 & 9 \\
\hline $\mathbf{7}$ & $\mathbf{A F 7}$ & 1500 & 1000 & 1.5 & 7 \\
\hline
\end{tabular}

Poly(azoanthraquinone)s

\begin{tabular}{|c|c|c|c|c|c|}
\hline & Name & $\mathbf{M}_{\mathbf{w}, \mathbf{G P C}}$ & $\mathbf{M}_{\mathbf{n}, \mathbf{G P C}}$ & $\mathbf{P D I}$ & $\mathbf{D P}_{\mathbf{w}}$ \\
\hline $\mathbf{1}$ & $\mathbf{A Q 1}$ & 3900 & 1650 & 2.4 & 17 \\
\hline $\mathbf{2}$ & $\mathbf{A Q 2}$ & 3150 & 1600 & 2.0 & 13 \\
\hline $\mathbf{3}$ & $\mathbf{A Q 3}$ & 2350 & 1200 & 1.9 & 10 \\
\hline $\mathbf{4}$ & $\mathbf{A Q 4}$ & 1400 & 800 & 1.7 & 6 \\
\hline $\mathbf{5}$ & AQ5 & 4300 & 1400 & 3.0 & 19 \\
\hline $\mathbf{6}$ & AQ6 & 3800 & 1300 & 3.0 & 16 \\
\hline
\end{tabular}

\section{Comment:}

In this publication, we demonstrate electrochemical properties of these new aromatic polymers. Molar mass distributions for obtained polymers are typical for a step growth polymerization, i.e. broad with a relatively low number of high molar mass products. The highest molar mass fraction was isolated using a quick column chromatography in amounts sufficient for characterization. This way we determined coupling conditions to pursue for polymerization. The more effective approach to synthesis of reported polymers and their isolation must be a subject of a separate publication. Authors realize that SEC only gives an estimate of the molar mass due to significant differences between flexible polymer standards and rigid polymers studied herein. However, optical methods are not appropriate due to light absorbance and intermolecular association. MALDI-TOF was not successful either. 


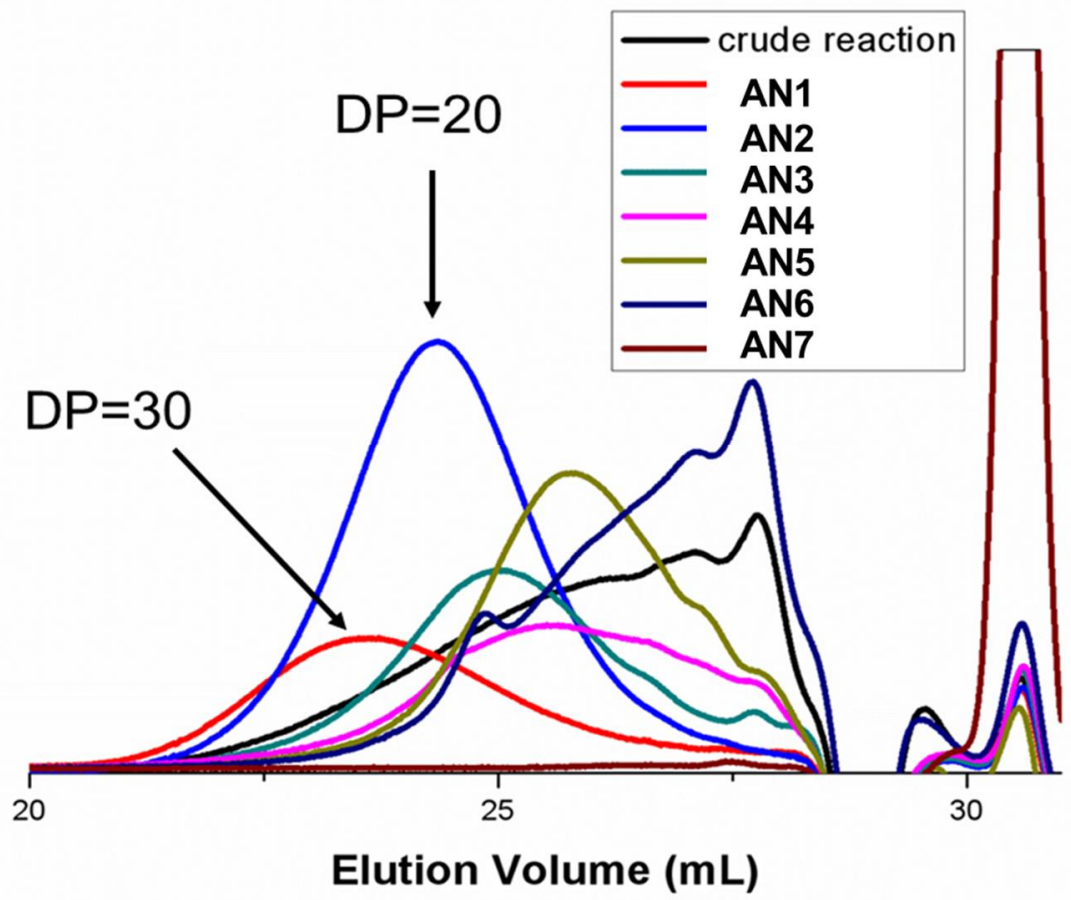

Fig. S4 Comparison of SEC Eluograms (THF) of poly(1,5-azonapthalene)s upon fractionation.

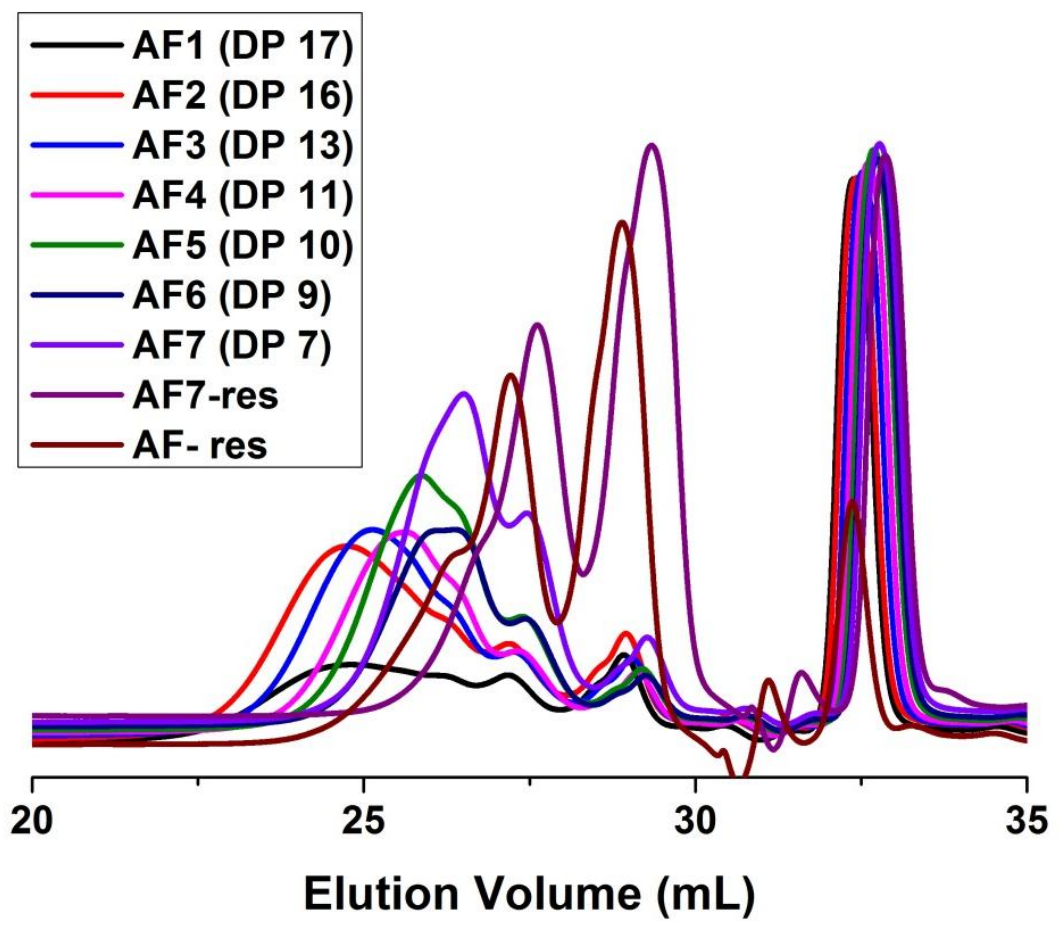

Fig. S5 Comparison of SEC Eluograms (THF) of poly(2,7-azofluorenone)s upon fractionation. 


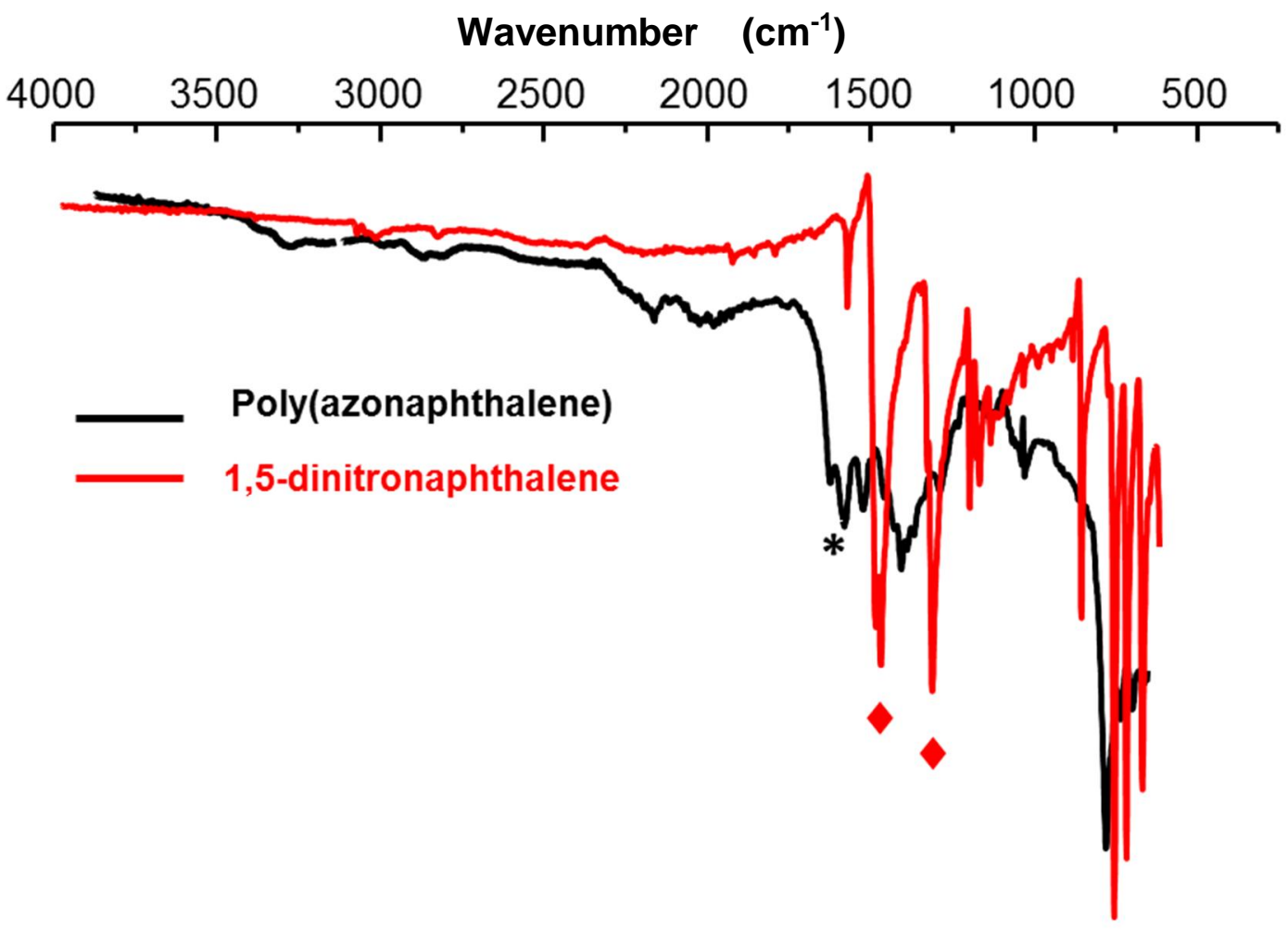

Fig. S6 Comparison of FTIR spectra of 1,5-dinitronaphthalene and the resulting polymers poly(azonaphthalene)s.

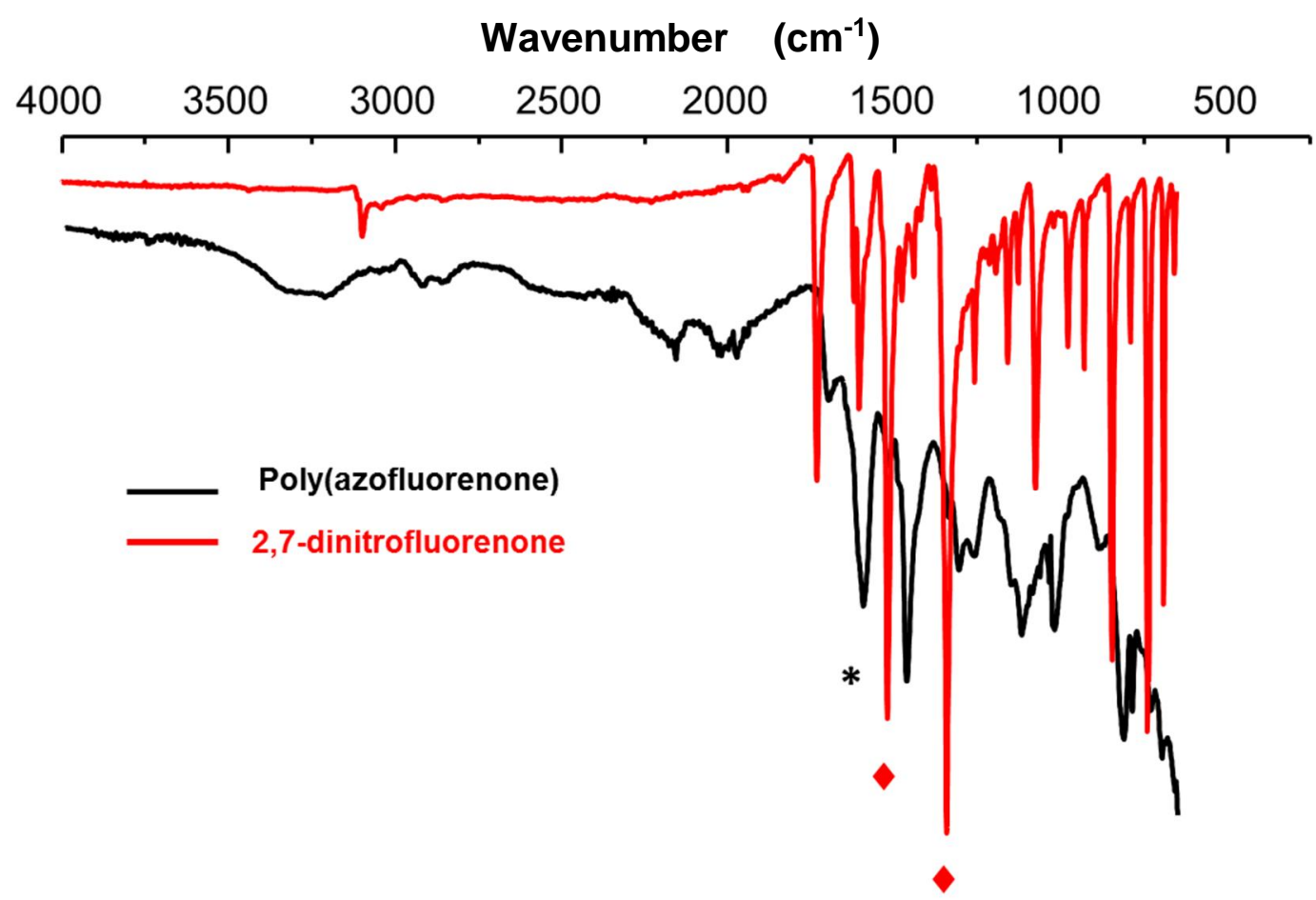

Fig. S7 Comparison of FTIR spectra of 2,7-dinitrofluorenone and the resulting polymers poly(azofluorenone)s. 


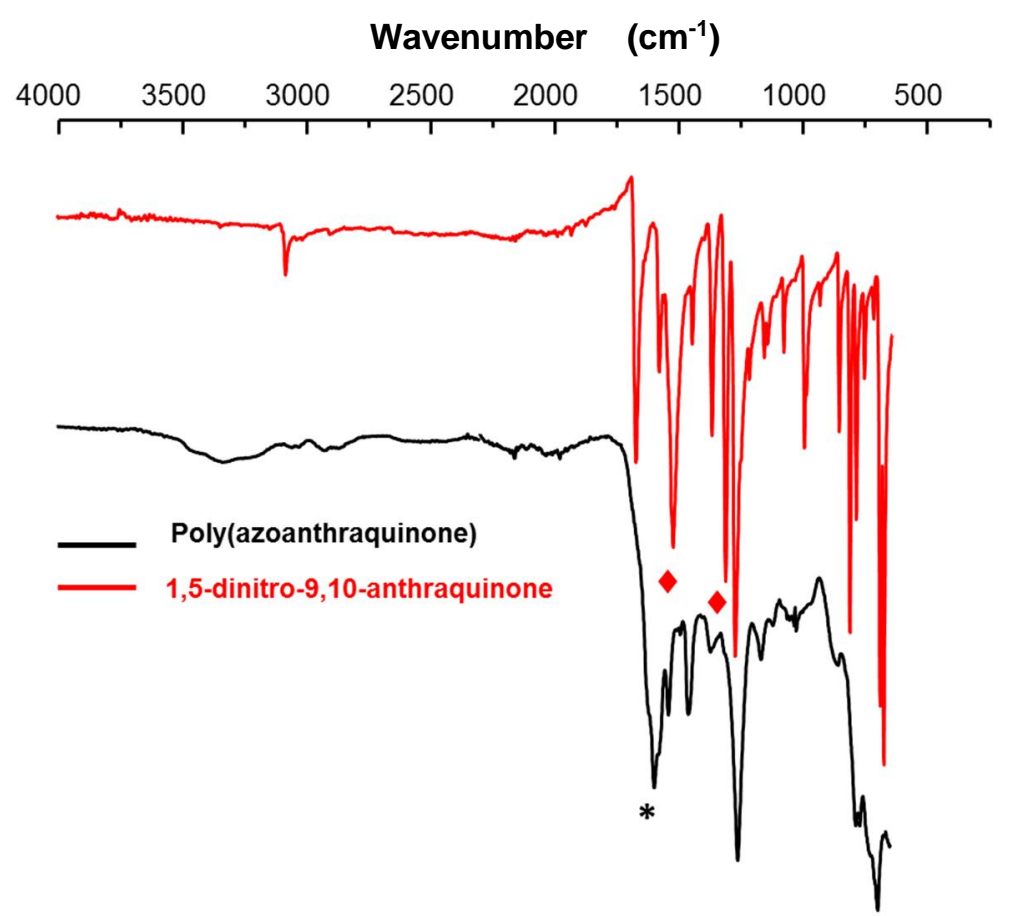

Fig. S8 Comparison of FTIR spectra of 1,5-dinitro-9,10-anthraquinone and the resulting polymers poly(azoanthraquinone)s.<smiles>[R]N=Nc1cccc2c(N=N[R])cccc12</smiles>
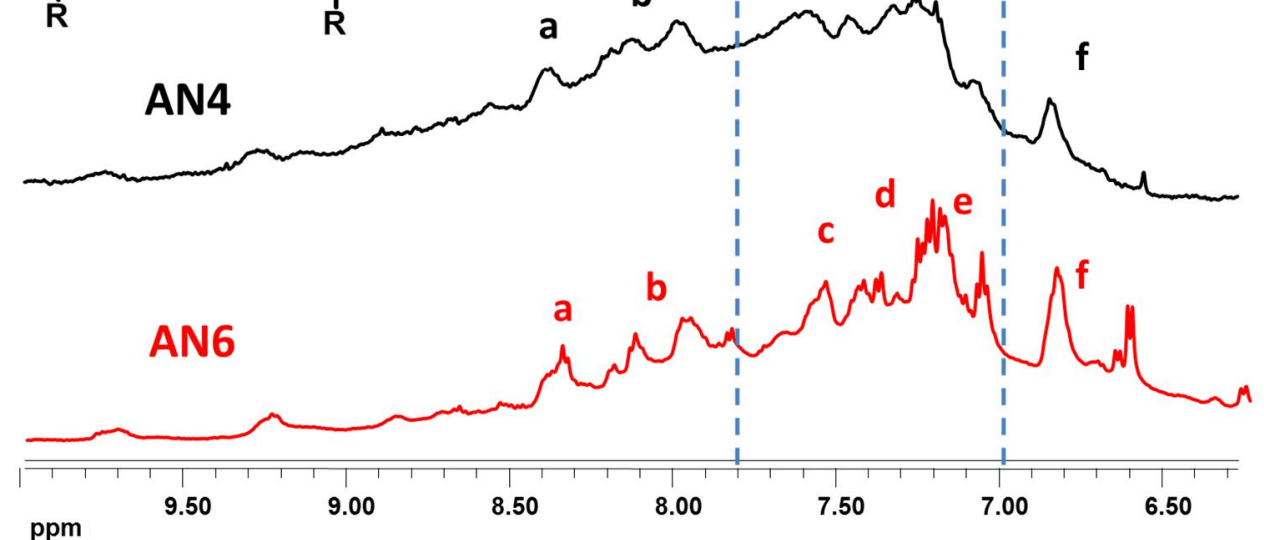

Fig. S9 Comparison of ${ }^{1} \mathrm{H}$ NMR spectra in DMSO-d6 (magnification 6.25 - 10ppm) of poly(azonaphthalene) fractions AN4 (DP 14) and AN6 (DP 7) highlighting the different characteristic regions of signals . 


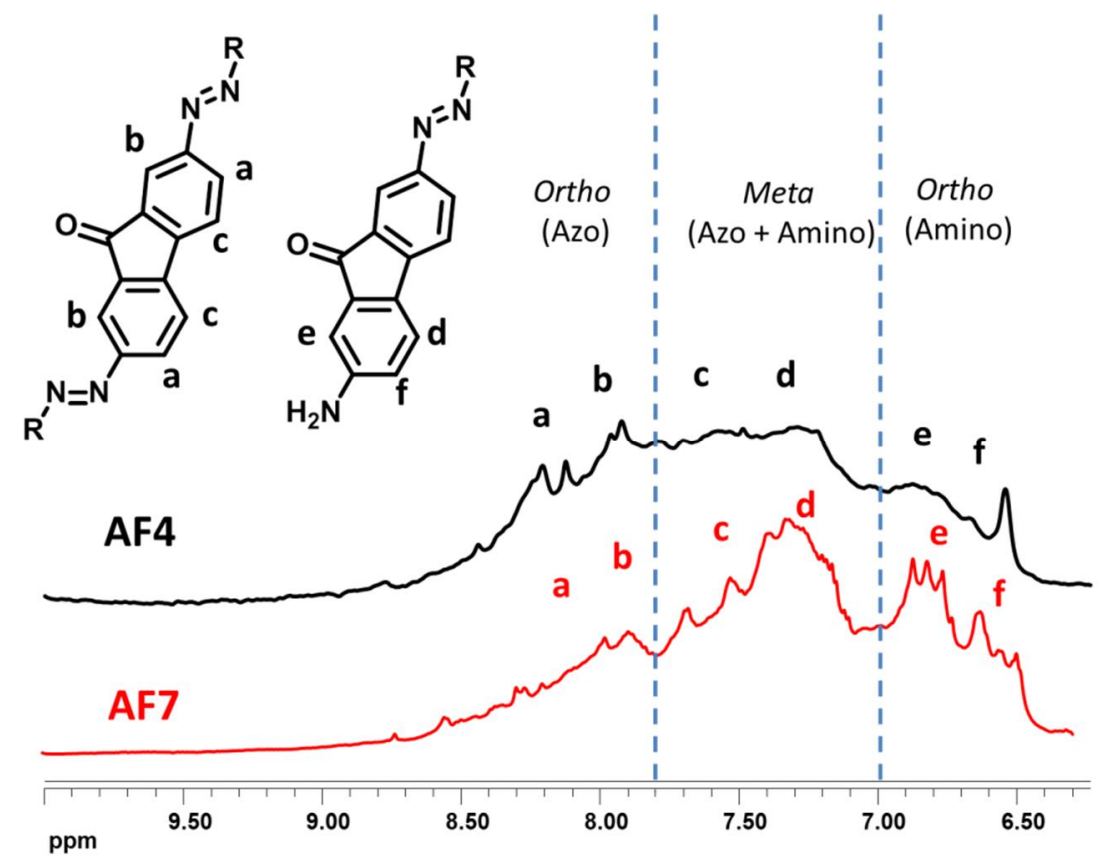

Fig. S10 Comparison of ${ }^{1} \mathrm{H}$ NMR spectra in DMSO-d6 (magnification 6.25 - 10ppm) of poly(azofluorenone) fractions AF4 (DP 11) and AF7 (DP 7) highlighting the different characteristic regions of signals .
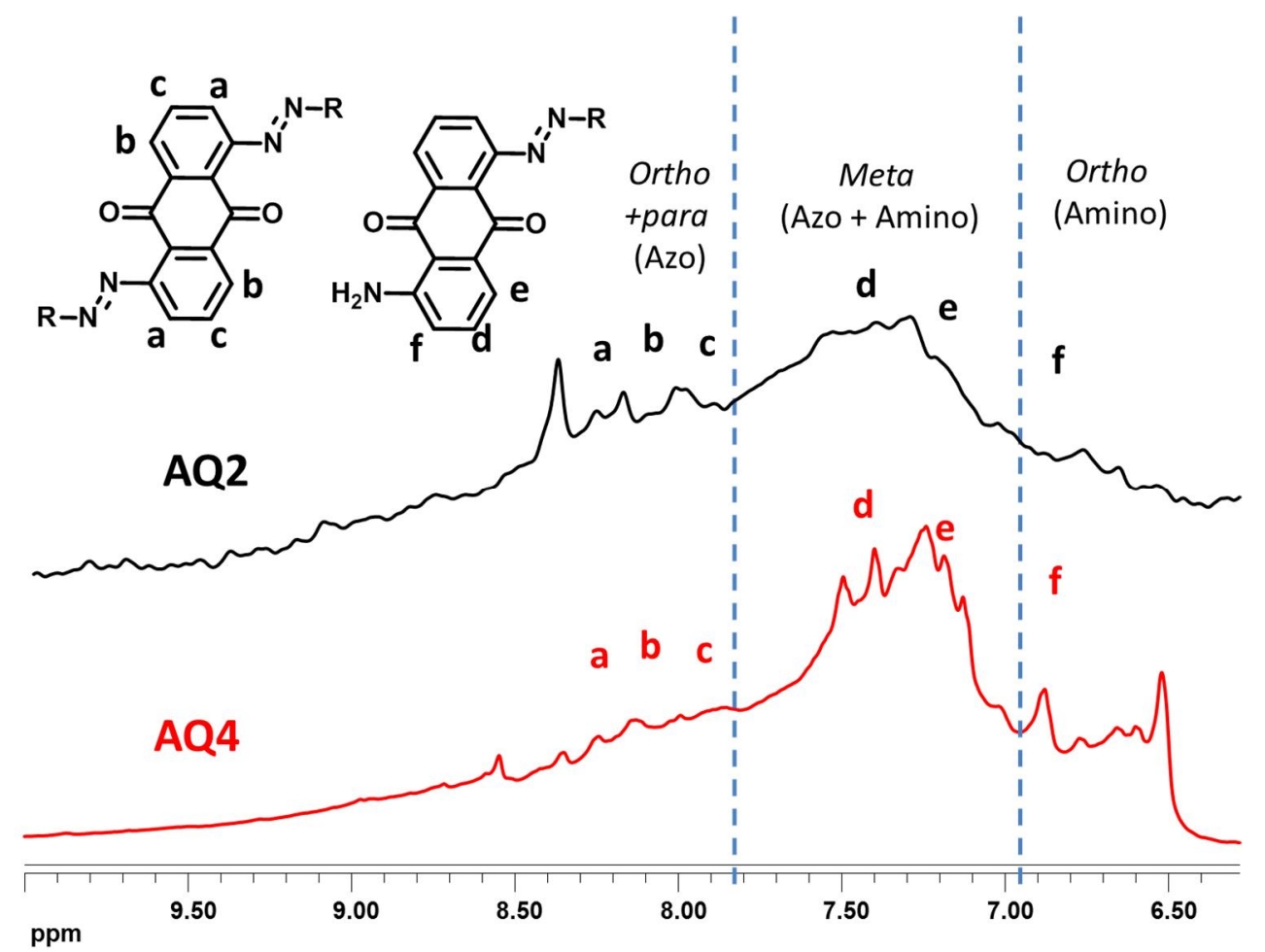

Fig. S11 Comparison of ${ }^{1} \mathrm{H}$ NMR spectra in DMSO-d6 (magnification 6.25 - 10ppm) of poly(azoanthraquinone) fractions AQ2 (DP 13) and AQ4 (DP 6) highlighting the different characteristic regions of signals. 

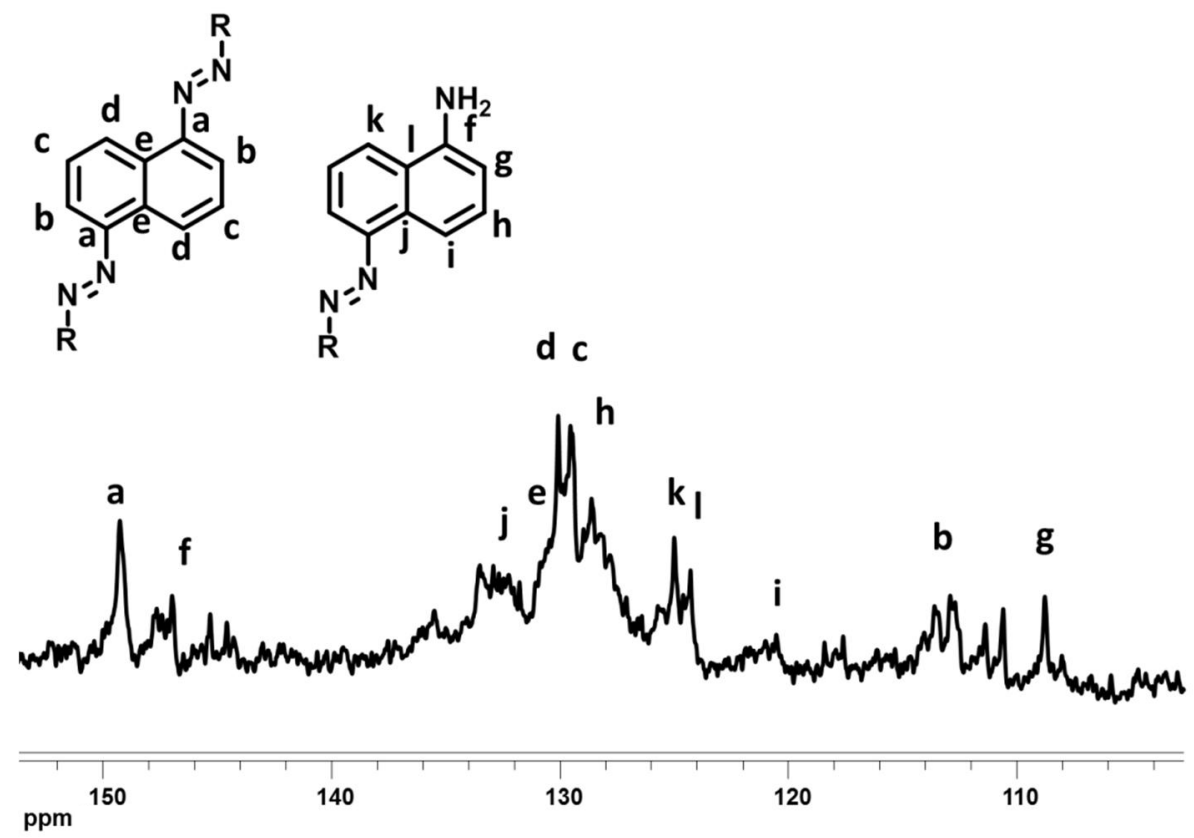

Fig. S12 Magnification (100-155 ppm) of ${ }^{13} \mathrm{C}$ NMR spectrum of poly(azonaphthalene) fraction AN6 (DP 7) in DMSO-d6 and assignment of signals<smiles>[R]N=Nc1ccc2c(c1)C(=O)c1cc(N=NN[R])ccc1-2</smiles>

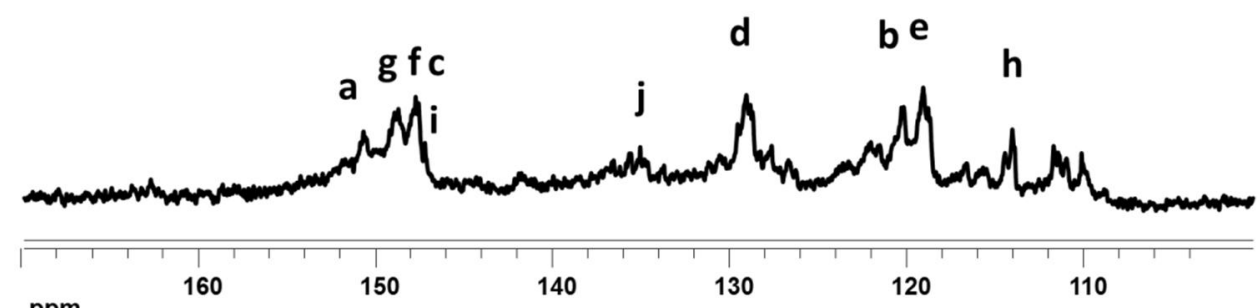

ppm

Fig. S13 Magnification (100-170 ppm) of ${ }^{13} \mathrm{C}$ NMR spectrum of poly(azofluorenone) fraction AF7 (DP 7) in DMSO-d6 and assignment of signals. 


\section{WILEY-VCH}
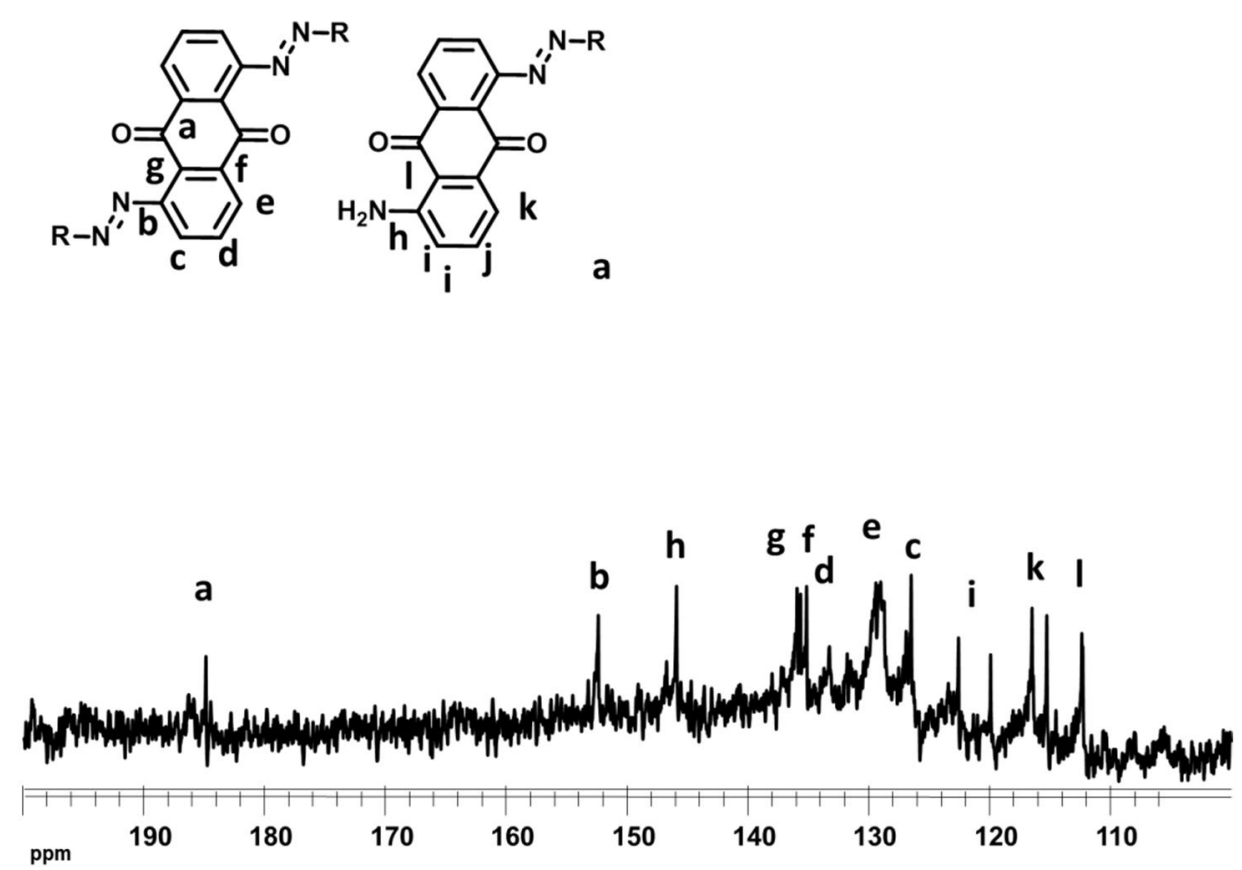

Fig. S14 Magnification (100-170 ppm) of ${ }^{13} \mathrm{C}$ NMR spectrum of poly(azoanthraquinone) fraction AQ4 (DP 6) in DMSO-d6 and assignment of signals. 


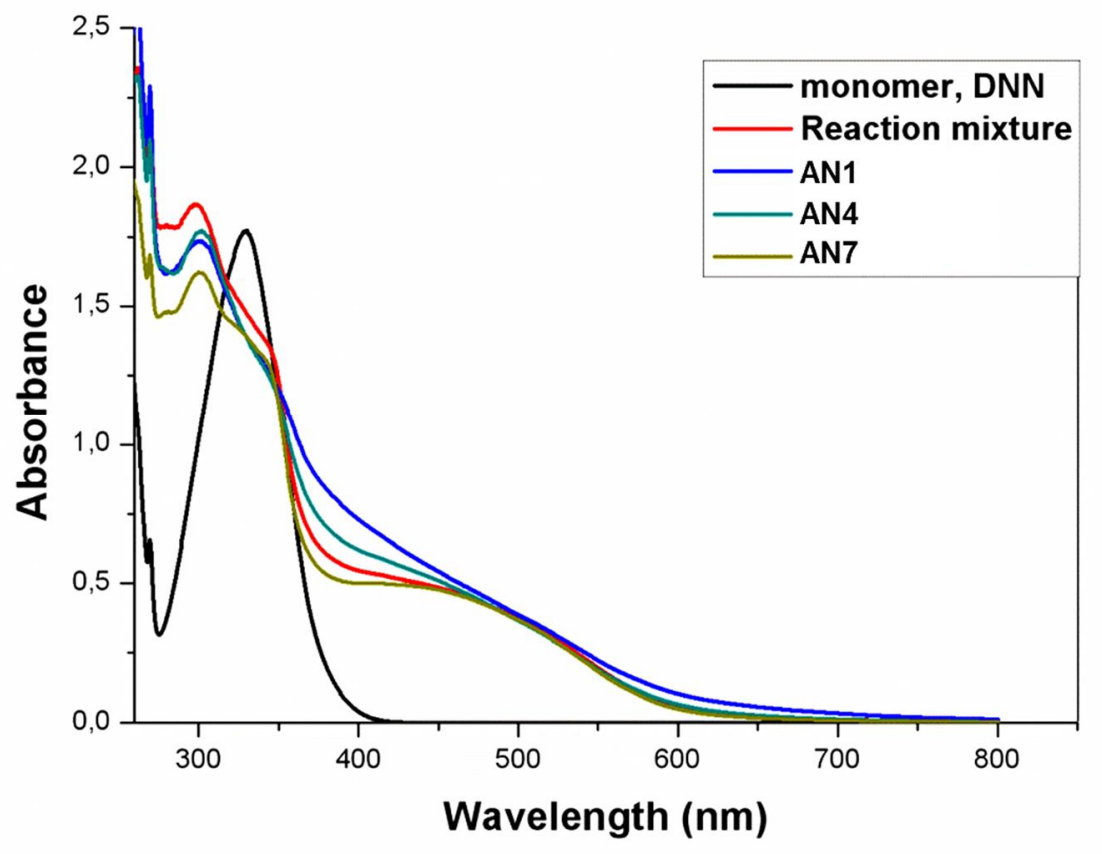

Fig. S15 UV Vis spectra of poly(1,5-azonaphthalene) fractions $(0.15 \mathrm{~g} / \mathrm{L}$ in THF $)$ depicting the dependence of absorbance on the degree of polymerization .

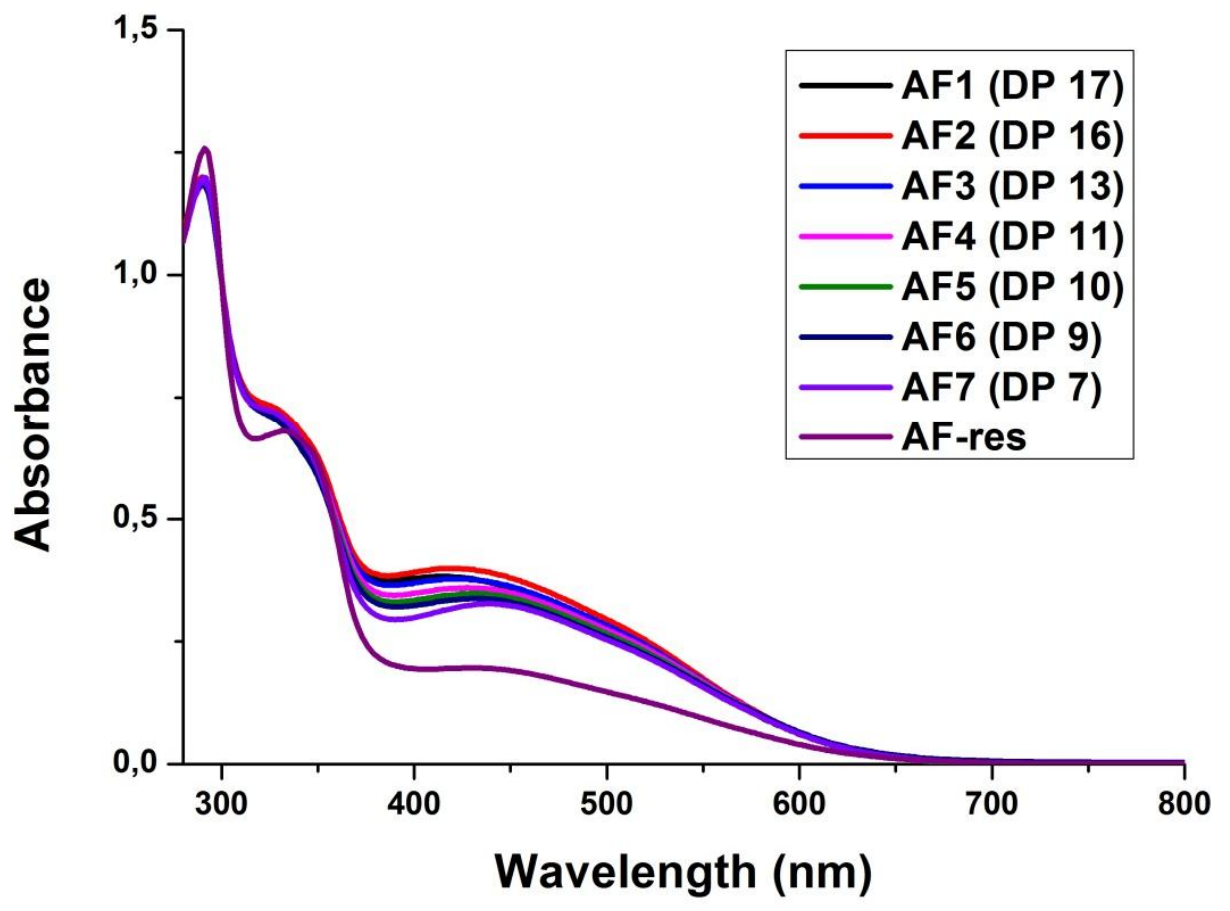

Fig. S16 UV Vis spectra of poly(2,7-azofluorenone) fractions $(0.1 \mathrm{~g} / \mathrm{L}$ in $5 \% \mathrm{DMF}$ in THF) depicting the dependence of absorbance on the degree of polymerization . 


\section{WILEY-VCH}

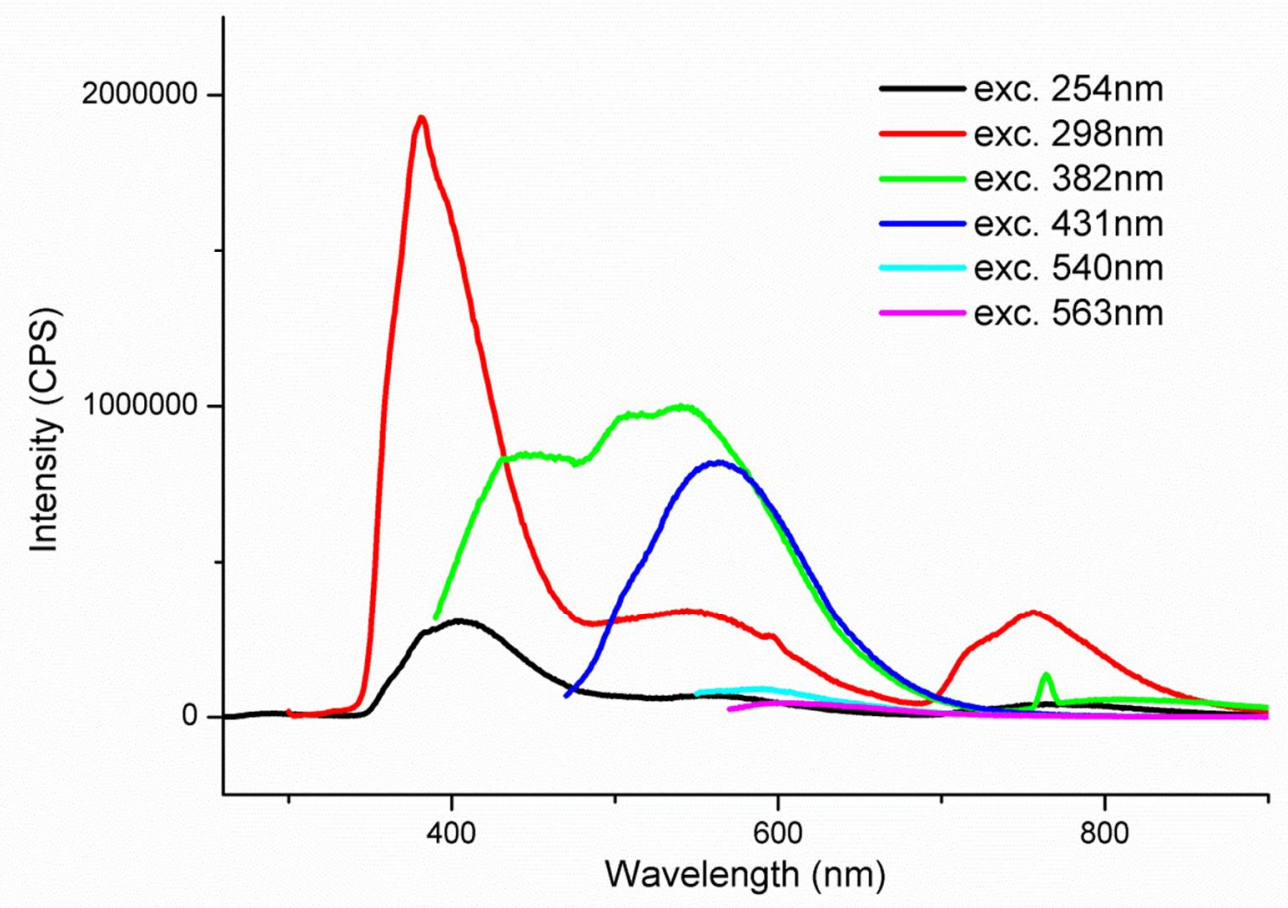

Fig. S17 Emission spectra of poly(1,5-azonaphthalene) fraction AN4 (DP=12) in THF (0.15 g/L) upon excitation with different wavelengths. 

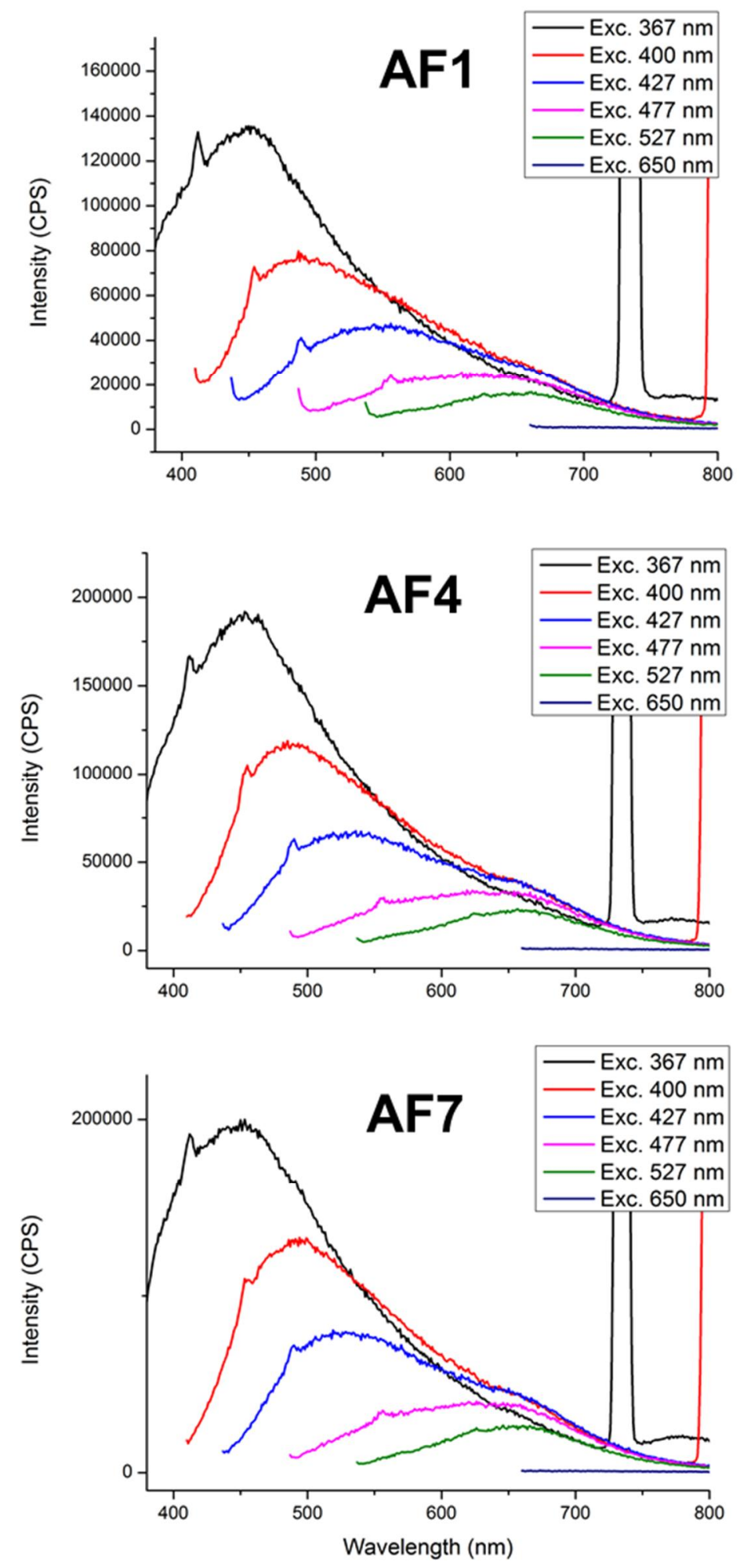

Fig. S18 Emission spectra of poly(2,7-azofluorenone) fraction AF1 (DP=16-17), AF4 (DP=10-11) and AF7 (DP 6-7) in 5\% DMF in THF (0.1g/L) upon excitation with different wavelengths. 

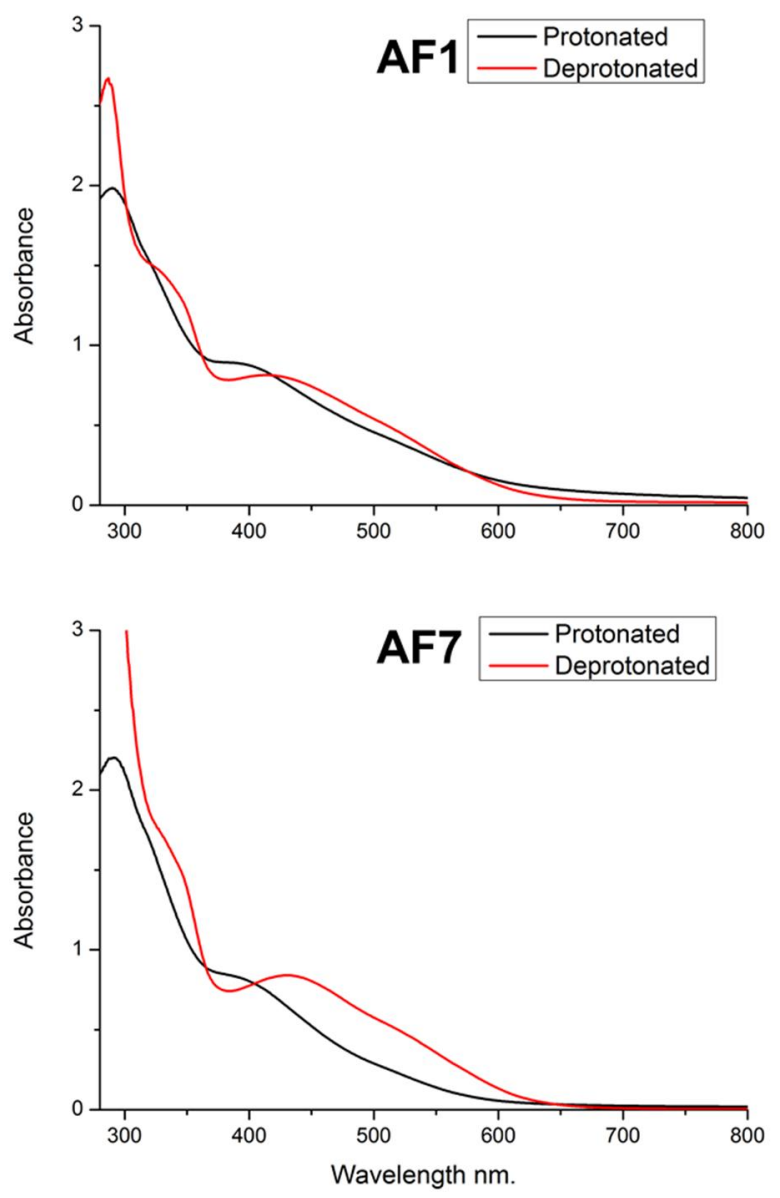

Fig. S19 UV Vis spectra of poly(2,7-azofluorenone) fractions AF1 (DP 16-17) and AF7 (DP=5-6) in the protonated and deprotonated state $(0.2 \mathrm{~g} / \mathrm{L}$ in $5 \% \mathrm{DMF}$ in THF).

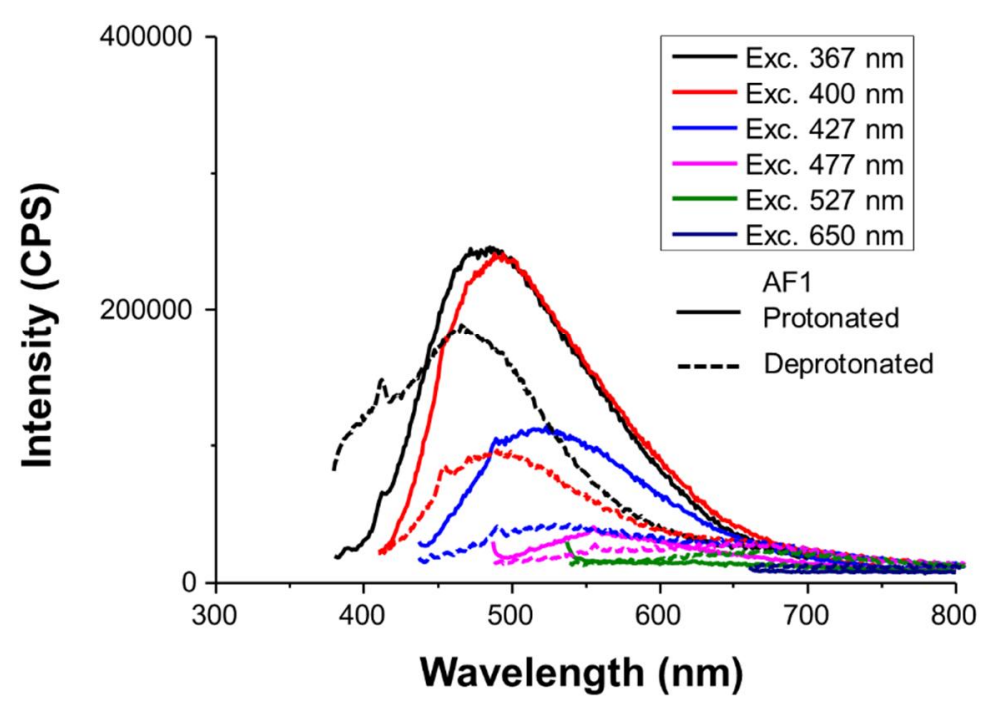

Fig. S20 Influence of protonation state on emission of the poly(azofluorenone) Fraction AF1 in 5\% DMF in THF $(c=0.2 \mathrm{~g} / \mathrm{L})$. 


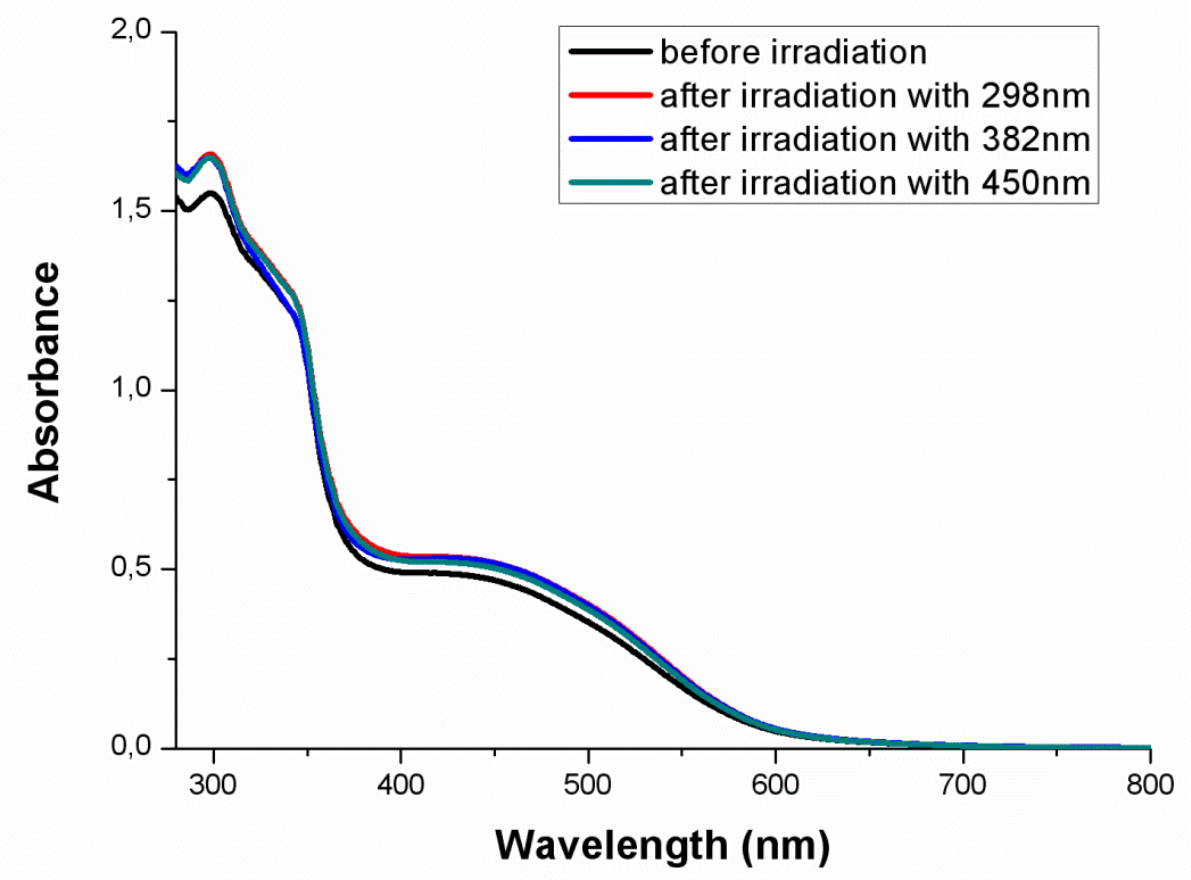

Fig. S21 UV Vis spectra of poly(1,5-azonaphthalene) fraction AN4 ( $0.1 \mathrm{~g} / \mathrm{L}$ in THF) before and after extended irradiation with different wavelengths. Time was several hours up to $12 \mathrm{~h}$. Power of light was 50$100 \mathrm{~mW}$.

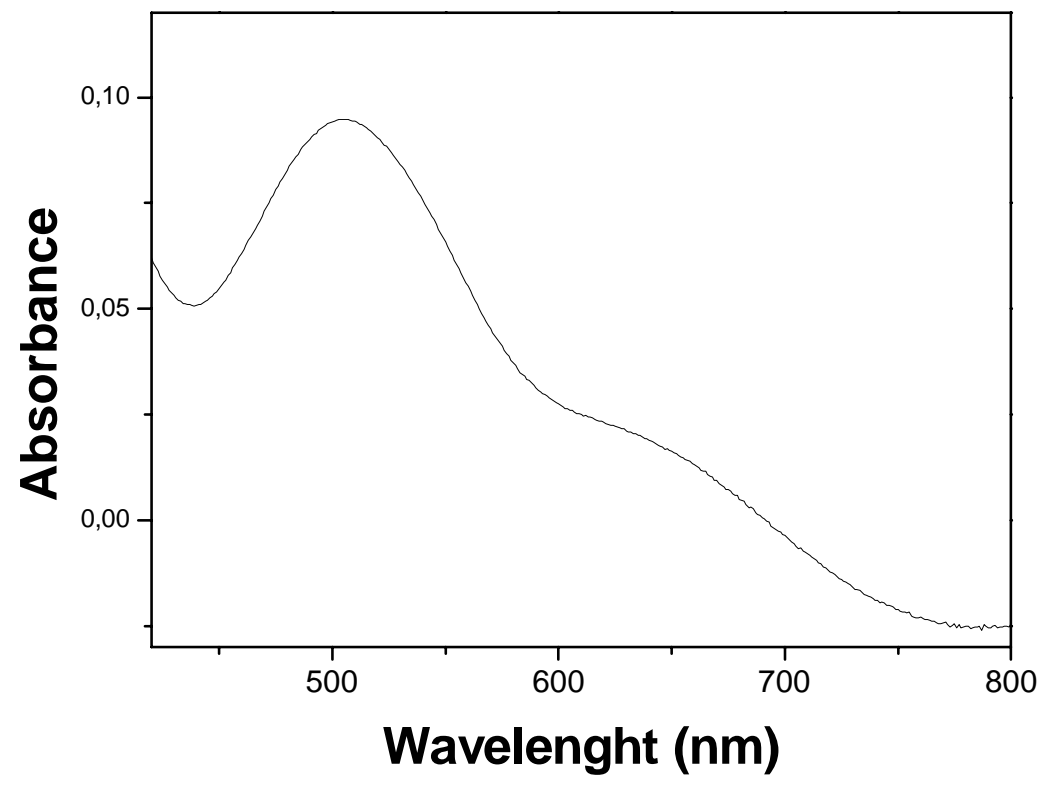

Fig. S22 UV-Vis spectrum of a poly(azoanthraquinone) film on an FTO substrate. 